\title{
A new European plant-specific emission inventory of biogenic volatile organic compounds for use in atmospheric transport models
}

\author{
M. Karl ${ }^{1, *}$, A. Guenther ${ }^{2}$, R. Köble ${ }^{1}$, A. Leip ${ }^{1}$, and G. Seufert ${ }^{1}$ \\ ${ }^{1}$ European Commission, Joint Research Centre, Institute for Environment and Sustainability, Ispra, Italy \\ ${ }^{2}$ National Center for Atmospheric Research, Boulder, CO, USA \\ *now at: NILU, Norwegian Institute for Air Research, Kjeller, Norway
}

Received: 10 September 2008 - Published in Biogeosciences Discuss.: 18 December 2008

Revised: 27 May 2009 - Accepted: 27 May 2009 - Published: 18 June 2009

\begin{abstract}
We present a new European plant-specific emission inventory for isoprene, monoterpenes, sesquiterpenes and oxygenated VOC (OVOC), on a spatial resolution of $0.089 \times 0.089$ degrees, for implementation in atmospheric transport models. The inventory incorporates more accurate data on foliar biomass densities from several litterfall databases that became available in the last years for the main tree species in Europe. A bioclimatic correction factor was introduced to correct the foliar biomass densities of trees and crops for the different plant growth conditions that can be found in Pan-Europe. Long-term seasonal variability of agriculture and forest emissions was taken into account by implementing a new growing season concept. The 20042005 averaged annual total biogenic volatile organic compound (BVOC) emissions for the Pan-European domain are estimated to be about $12 \mathrm{Tg}$ with a large contribution from the OVOC class of about $4.5 \mathrm{Tg}$ and from monoterpenes of about $4 \mathrm{Tg}$. Annual isoprene emissions are found to be about $3.5 \mathrm{Tg}$, insensitive to the chosen emission algorithm. Emissions of OVOC were found to originate to a large extent from agriculture. Further experiments on crop emissions should be carried out to check the validity of the applied standard emission factors. The new inventory aims at a fully transparent and verifiable aggregation of detailed land use information and at the inclusion of plant-specific emission data. Though plant-specific land use data is available with relatively high accuracy, a lack of experimental biomass densities and emission data on terpenes, sesquiterpenes and oxygenated VOC, in particular for agricultural plants, currently limits the setup of a highly accurate plant-specific emission inventory.
\end{abstract}

Correspondence to: G. Seufert (guenther.seufert@jrc.it)

\section{Introduction}

The amount and composition of plant species that cover the land surface is the primary control on the type and magnitude of biogenic volatile organic compound (BVOC) flux. On a shorter time scale, variation in atmospheric conditions determines the amount of photosynthetically active photon flux density (PPFD) reaching the leaf surface, the leaf temperature, and soil moisture. All these factors in turn influence variations in BVOC emissions. Temperature and light intensity are key driving variables that regulate emissions of isoprene, monoterpenes and other BVOCs in published emission algorithms (Tingey et al., 1991; Guenther et al., 1993, 1995, 2006; Schuh et al., 1997; Staudt et al., 2000).

Once emitted to the atmosphere, BVOCs react in the presence of nitrogen oxides to increase the concentration of tropospheric ozone (Atkinson and Arey, 2003), which is a respiratory irritant and major component of smog. Emissions of BVOC are significant to ozone production in the European boundary layer (Simpson, 1995; Vogel et al., 1995). The oxidation products of terpenes condense to form secondary organic aerosols (Hoffmann et al., 1997; Kavouras et al., 1998; Griffin et al., 1999) which directly alter Earth's radiative balance and can serve as cloud-condensation nuclei (Andreae and Crutzen, 1997). On the basis of results from smog chamber experiments of Pandis (1991) it has commonly been assumed that the photooxidation of isoprene does not contribute to the production of secondary organic aerosol under ambient conditions (Seinfeld and Pandis, 1998). However, evidence from both field and laboratory experiments has been obtained in the last four years for isoprene contributing to SOA formation (Claeys et al., 2004; Kourtchev et al., 2005; Kroll et al., 2005). Sesquiterpenes are a highly reactive BVOC class with atmospheric lifetimes of only a few minutes. These compounds have a high potential to form

Published by Copernicus Publications on behalf of the European Geosciences Union. 


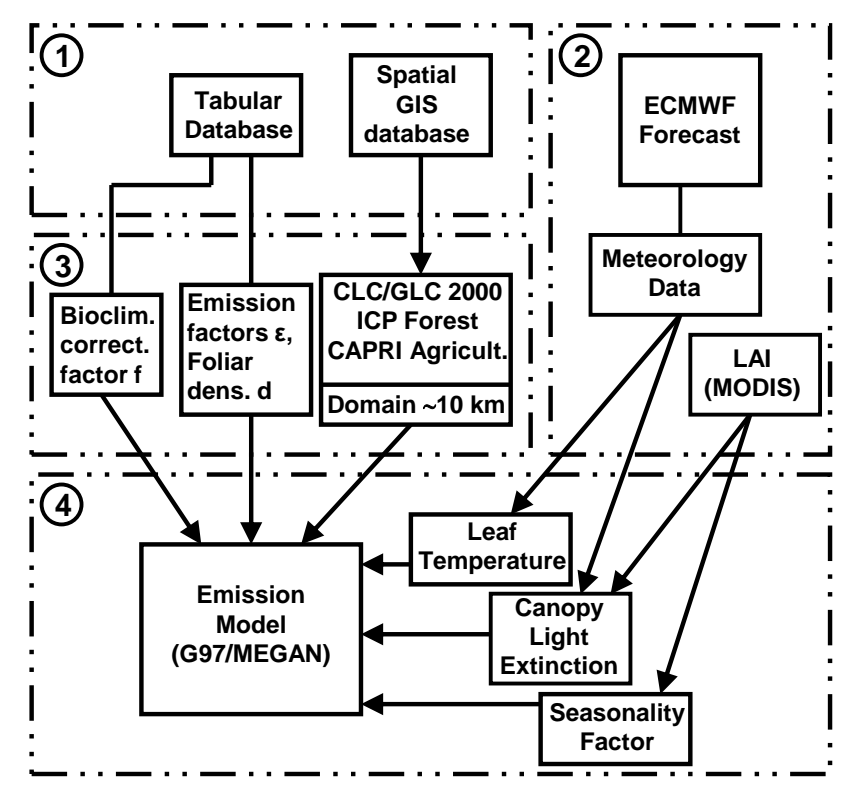

Fig. 1. BVOC emission model, schematic overview.

secondary organic aerosol (Hoffmann et al., 1997; Jaoui et al., 2003). Bonn and Moortgat (2003) suggest that the reaction of sesquiterpenes with atmospheric ozone could be responsible for the atmospheric new particle formation observed frequently in rural locations.

Recent observations in boreal forests support the role of aerosol production from biogenic hydrocarbon precursors in the activation of cloud droplets (Kerminen et al., 2005; Tunved et al., 2006). In addition, BVOCs released to the atmosphere may represent a relevant source term in the overall carbon budget of an ecosystem (Guenther, 2002; Kesselmeier et al., 2002).

The European landscape is characterised by a great variety of climatic and orographic zones and biomes, ranging from boreal forests in Scandinavia and Russia to Mediterranean shrub vegetation. For millennia, the European land cover has been intensively modified by man to create an extremely patchy landscape. In addition the orography of Europe is complex, with changes between mountainous and plain landscapes on a small scale. Type and amount of BVOC emissions is highly variable; even within a plant taxonomic group like the important Quercus (oak) genus one can observe several species emitting huge amount of isoprene, other species emitting monoterpenes, others are close to zero emitters (Csiky and Seufert, 1999). Therefore it is of great importance to use highly resolved land cover data of forests, agriculture and other land use for modelling European BVOC emissions.

Estimates of European isoprene emission depend on the choice of emission algorithms describing the temperature and light dependence of emissions. Two different representations of the temperature and light dependence, G97 (Guen- ther, 1997) and MEGAN (Guenther et al., 2006), were used to calculate emission fluxes of isoprene over Europe. Previous European emission estimates for isoprene were about $4 \mathrm{Tg}$ per year, and for total BVOC ranged between $7.5 \mathrm{Tg}$ to 29 Tg per year (Lübkert and Schöpp, 1989; Andryukov and Timofeev, 1989; Simpson et al., 1999; Steinbrecher et al., 2009), indicating the high uncertainty of BVOC inventories. The reason for this high variability is not always easy to understand, because inventories are not always fully transparent or cannot be compared for various reasons. Therefore, the new inventory presented here aims at full transparency and avoidance of unnecessary complexity in order to allow calculations in the frame of a global chemical transport model.

\section{Inventory description}

In this work a new plant-specific BVOC emission inventory with high spatial resolution for implementation in atmospheric transport models is developed for Europe. Data on emission potentials at standard conditions for tree and crop species and for landcover classes was adopted from the work of Steinbrecher et al. (2009), the NatAir (Improving and Applying Methods for the Calculation of Natural and Biogenic Emissions and Assessment of Impacts on Air Quality) project inventory of VOC emission from natural and semi-natural vegetation in Pan-Europe. Throughout this work, emission potentials are given for standard conditions, i.e. $30^{\circ} \mathrm{C}$ leaf temperature and $1000 \mu \mathrm{mol} \mathrm{m}^{-2} \mathrm{~s}^{-1}$ photosynthetic photon flux density (PPFD). From several litterfall databases we derived more accurate data on foliar biomass densities for the most abundant tree species. A biomass correction factor was introduced for forest and agriculture land use classes to adjust foliar biomass densities for growing conditions in different climates. To account for the long-term seasonal variability of emissions, a new growing season concept was introduced based on detailed phenology data for major agricultural crops and on a seasonal algorithm for deciduous and evergreen vegetation by Staudt et al. (2000).

For each BVOC class the emissions can be calculated as sum over all grid cells of the European domain:

$E_{b}=\sum_{i} A_{i} \cdot \epsilon_{b, i} \cdot D_{i} \cdot \gamma_{\mathrm{CE}, i}$

In Eq. (1), $A_{i}$ is the vegetated and emitting area in a grid cell $i$ (in $\left.\mathrm{m}^{2}\right), \epsilon_{b, i}$ is the average emission standard potential (in $\mu \mathrm{g} \mathrm{g}_{\mathrm{DW}}^{-1} \mathrm{~h}^{-1}$ ) of each BVOC class, $D_{i}$ the foliar biomass density related to dry mass (in $\mathrm{g} \mathrm{m}^{-2}$ ) and $\gamma_{\mathrm{CE}, i}$ is the dimensionless canopy emission activity factor, often referred to as the environmental correction factor.

The schematic information flow of our BVOC emission model is depicted in Fig. 1. The model contains four main parts: (1) retrieving geo-referenced spatial data from several Geographic Information System (GIS) databases and property data from tabular databases, (2) meteorological 
input data from the European Centre for Medium Range Weather Forecast (ECMWF) and leaf area index data from MODIS, (3) aggregation of the spatial GIS data for the PanEuropean domain, processing of tabular input and writing output to HDF files, (4) calculation of BVOC emissions using a Canopy Environment Model written in Interactive Data Language (IDL). In Sects. 2.1 to 2.3 the procedure of retrieving and aggregating spatial information with plant specific properties is described in detail (part 1 and 3 in Fig. 1). Section 2.4 describes the Canopy Environment Model which was applied to calculate the emission activity factor $\gamma_{\mathrm{CE}, i}$ in Eq. (1). Throughout the paper, emission factors and emission rates refer to mass of BVOC compound. We note that isoprene emission estimates by Guenther et al. (2006) are given in mass carbon and that these were converted to mass of isoprene for the comparison with the estimates of this work (Sect. 3.5).

\subsection{Land use aggregation}

In this section we derive the vegetated and emitting area $A_{i}$ in a grid cell. A new land use map covering the European continental domain $\left(-20^{\circ} \mathrm{W}, 20^{\circ} \mathrm{N}\right.$ to $\left.40^{\circ} \mathrm{E}, 72^{\circ} \mathrm{N}\right)$ is compiled by combination of tree species distributions from the Tree Species Map (Köble and Seufert, 2002) and crop species distributions from the Agricultural Land Use Map (Leip et al., 2008) with the CLC/GLC2000 mosaic (Köble, 2007) land cover data. The Tree Species Map distinguishes 115 tree species and is based on the inventory of the ICP (International Cooperative Programme on Assessment and Monitoring of Air Pollution Effects on Forests) Forest Focus level 1 network. The Agricultural Land Use Map includes 26 crop species and is based on work of the project CAPRI-DynaSpat (Common Agricultural Policy Regional Impact Analysis The Dynamic and Spatial Dimension). The Agricultural Land Use Map contains crop-specific information for EU15 and the Tree Species Map contains tree-specific information for 30 European countries. According to Köble and Seufert (2002), the main tree species in Europe are Pinus sylvestris with a percentage contribution of $31.2 \%$ to the forested area in 30 countries, followed by Picea abies (21.4\%), Fagus sylvatica (7.1\%), Betula spp. (7.0\%), Quercus robur (2.9\%), Pinus pinaster (2.5\%), Quercus petraea $(2.3 \%)$.

The CLC/GLC2000 mosaic contains 50 vegetation classes, combining the Corine Land Cover map CLC2000 (see http://terrestrial.eionet.eu.int/) and the global and different regional versions of the GLC2000 (http://bioval.jrc.ec. europa.eu/products/glc2000/glc2000.php) for the European continent. Vegetation classes from the Global Land Cover, GLC2000, were divided into Africa, Northern Eurasia, and Europe parts, if their geographical distribution covers different parts of Pan-Europe. The African region corresponds to the European part of the GLC Africa v5.0 and the GLC Global v1.1, the Northern Eurasia region to the European part of GLC Northern Eurasia v4.0 and the Europe region corresponds to the extent of GLC Europe v1.0.

All land use data was aggregated and then projected on a regular latitude/longitude grid. The data is available in WGS84 projection with a horizontal resolution of $0.089 \times 0.089$ degrees for continental Pan-Europe. Since longitudes are widest at the equator and converge towards the poles, the grid cell area in the new latitude/longitude grid corresponds to about $9.6 \mathrm{~km}$ by $9.6 \mathrm{~km}$ at the southern border $\left(20^{\circ} \mathrm{N}\right)$ and to about $9.6 \mathrm{~km}$ by $3.2 \mathrm{~km}$ at the northern border $\left(72^{\circ} \mathrm{N}\right)$ of the domain.

In the equations below, $t$ is used as an index for tree species, $c$ for crop species, $g$ is an index for the general CLC/GLC 2000 land use classes, and $b$ denotes the BVOC classes.

The CLC/GLC2000 mosaic land use map covers the complete Pan-European domain and the sum of area fractions of CLC/GLC2000 mosaic vegetation classes $\left(a_{\mathrm{clc}, i}\right)$ in a grid cell $i$ is assumed to be the total vegetated area fraction. General land use classes from the CLC/GLC2000 mosaic belong to one of the three main land use categories: agriculture, forest and other land use category (i.e. shrubland, wetland, and grassland). The CLC/GLC2000 area fraction in a grid cell is the sum of the cumulated area fractions of agriculture, $a_{\mathrm{clcagr}, i}$, forest, $a_{\mathrm{clcfor}, i}$, and other land use, $a_{\mathrm{clcoth}, i}$, vegetation classes:

$a_{\mathrm{clc}, i}=a_{\mathrm{clcagr}, i}+a_{\mathrm{clcfor}, i}+a_{\mathrm{clcoth}, i}$

By relating the cumulated areas of the CLC/GLC2000 mosaic to the total area in each grid cell, it was assured that $a_{\mathrm{clc}, i}$ does not exceed 1 in each grid cell. Thus the vegetated areas provided by the CLC/GLC2000 mosaic can be used as a reference for the plant-specific land use data from the Tree Species Map and from the Agricultural Land Use Map. The advantage of using the CLC/GLC2000 mosaic as a reference is that it covers the complete Pan-European region, while both the Tree Species Map and the Agricultural Land Use Map cover only parts of the European domain.

In the next step, forest and agriculture areas of the CLC/GLC2000 mosaic land cover in a cell were replaced with the areas of the respective tree and crop species from the Tree Species Map and the Agricultural Land Use Map, where this information was available. The forested area in a grid cell covered with the Tree Species Map should be the same as the area from forest vegetation classes defined in the CLC/GLC2000 mosaic. But since the maps were created using different methods and data sources, there may be inconsistencies between the CLC/GLC2000 mosaic and the plant-specific land use maps. To avoid these inconsistencies in the new European map, the area fraction $a_{t, i}$ for a single tree species $t$ and the area fraction $a_{c, i}$ for a single crop species $c$ is corrected with the cumulated area fraction of CLC/GLC2000 forest or agriculture vegetation 
classes within a grid cell. The corrected area fraction of a tree species, $a_{t, i}^{\prime}$, is:

$a_{t, i}^{\prime}=a_{t, i} \cdot \frac{a_{\mathrm{clcfor}, i}}{\sum_{t} a_{t, i}}$

and the corrected area fraction of a crop species, $a_{c, i}^{\prime}$, is:

$a_{c, i}^{\prime}=a_{c, i} \cdot \frac{a_{\mathrm{clcagr}, i}}{\sum_{c} a_{c, i}}$

The grid cell area $A_{i}\left(\right.$ in $\mathrm{m}^{2}$ ) (see Eq. 1), that is covered with vegetation and emits $\mathrm{BVOC}$ is:

$A_{i}=\left(\sum_{t, i} a_{t, i}^{\prime}+\sum_{c, i} a_{c, i}^{\prime}+a_{\mathrm{clcoth}, i}\right) \cdot A_{\mathrm{cell}, i}$

where $A_{\text {cell, } i}$ is the total area of the grid cell $i\left(\right.$ in $\left.^{2}\right)$.

\subsection{Foliar biomass density}

Foliar biomass density distribution for Europe is determined from the land use information and plant-specific values. The foliar biomass density $D_{i}$ in a grid cell (see Eq. 1) is the sum of the foliar densities of trees, $d_{t}$, and of crops, $d_{c}$, in the cell, weighted by the area contribution of the plant to the cell area. For the remaining area of a grid cell, covered with vegetation of CLC/GLC2000 classes, the foliar density $d_{g}$ is added, weighted by the area fraction $a_{g, i}$ of the CLC/GLC2000 class $g$ in that cell:

$D_{i}=\sum_{t} a_{t, i}^{\prime} d_{t} f_{t}+\sum_{c} a_{c, i}^{\prime} d_{c} f_{c}+\sum_{g} a_{g, i} d_{g} f_{g}$

where $f_{t}, f_{c}$ and $f_{g}$ are the dimensionless zonal bioclimatic correction factors for trees, crops and CLC/GLC2000 vegetation classes, respectively. European foliar biomass density distributions calculated from Eq. (6) for agriculture, forest, other land use and total land use are shown in Fig. A1 in the Electronic Supplement (see http://www.biogeosciences.net/ 6/1059/2009/bg-6-1059-2009-supplement.pdf).

To consider different growth conditions for the same tree species within Pan-Europe, e.g. in the Boreal zone compared to growth conditions in Central Europe, the foliar biomass densities were scaled relative to growing conditions in the continental vegetation zone. For this purpose we introduced zonal bioclimatic correction factors for the different plant species and vegetation classes. Zonal bioclimatic correction factors were defined for 10 European vegetation zones using the Indicative Map of European Biogeographical Regions developed by the European Environment Agency (2007): Alpine, Boreal, Atlantic, Continental, Steppic, Pannonian, Anatolian, Mediterranean, Black Sea and Africa-Arabia. The Africa-Arabia zone extends over Northern Africa, Western Arabia and the biogeographic region Macaronesia (Canary Islands, Madeira and Azores Islands).
The Continental vegetation zone is used as a reference zone to define bioclimatic correction factors for trees $\left(f_{t}\right)$, crops $\left(f_{c}\right)$, and CLC/GLC2000 vegetation classes $\left(f_{g}\right)$. Table 1 lists bioclimatic correction factors for main tree species and Table 2 for crop species in each vegetation zone.

To derive the biomass correction of a vegetation zone of interest, tree foliar biomass data and agricultural biomass data for both the selected vegetation zone and the Continental vegetation zone is needed. Tree and forest foliar biomass data was derived from litterfall and foliar net primary productivity (NPP) data and crop and agriculture biomass data was derived from national crop yield information. The zonal bioclimatic factor for a tree species is defined as the ratio of the average foliar biomass of this tree species in the selected vegetation zone to its average foliar biomass in the Continental zone. The zonal bioclimatic factor for a crop species is defined as the ratio of the harvested yield of this crop in the selected vegetation zone to its crop yield in the Continental zone. The extraction of the necessary tree foliar biomass data and crop yield data from available databases is described in the following.

Litterfall data and foliar NPP are used as indicators for growth conditions for the main tree species. The information on the litterfall and foliar NPP of trees at European sites is extracted from four databases: (1) leaf fall data from the global litterfall database of the Oak Ridge National Laboratory (ORNL) Distributed Active Archive Center (DAAC) (Holland et al., 2005), (2) the Forest Focus database for the ICP level2 plots (http://forest.jrc.ec.europa. eu/; http://www.icp-forests.org/), (3) the foliage component of NPP from the recent global database on forest carbon fluxes and pools presented by Luyssaert et al. (2007), (4) leaf fall data for birch (Betula spp.) from the new AFOLU (Agriculture, Forestry and Other Land Uses) Biomass Compartments database (Teobaldelli, 2008, see http://afoludata.jrc. ec.europa.eu/index.php/login). Annual dry foliar biomass in $\mathrm{g} \mathrm{m}^{-2}$ was calculated for each data record at European sites from the four databases. NPP data for the foliar compartment from Luyssaert et al. (2007) were converted from carbon to dry biomass using a conversion factor of 2 . We note that the conversion factor from dry biomass to carbon may vary depending on the actual $\mathrm{C} / \mathrm{N}$ ratio (Holland et al., 2005) but due to incomplete datasets a factor of 2 was generally used for the conversion. Depending on the geographic location of the forest site, the resulting foliar biomass for a certain tree species was attributed to the corresponding vegetation zone. By this procedure, altogether 89 litterfall records were compiled for Pinus sylvestris, 119 for Picea abies, 54 for Fagus sylvatica, 439 for Betula spp., and 92 for Quercus robur and Quercus petraea. Zonal bioclimatic correction factors for tree species and forests are given in Table 1.

Crop yields are used as an indicator for growth conditions of crops. National crop yields per hectare were obtained from the Food and Agriculture Organization of the United Nations (FAO) (FAOSTAT, 2007), for all countries within 
Table 1. Bioclimatic correction for main tree species and forest vegetation classes in European vegetation zones. The Continental vegetation zone is used as the reference zone $\mathrm{a}^{\mathrm{a}, \mathrm{b}}$.

\begin{tabular}{llrrrrrrr}
\hline & Tree species & Alpine & Arctic & Atlantic & Boreal & Contin. & Mediterr. & Steppic \\
\hline & Betula spp. & 0.81 & 0.51 & 0.73 & 1.36 & 1.00 & 1.00 & 1.11 \\
& Fagus sylvatica & 0.73 & 0.73 & 0.74 & 0.73 & 1.00 & 1.00 & 1.00 \\
& Picea abies & 0.86 & 0.60 & 0.58 & 0.60 & 1.00 & 1.00 & 1.00 \\
& Pinus sylvestris & 0.96 & 0.96 & 1.83 & 0.96 & 1.00 & 1.69 & 2.09 \\
& Quercus robur/petraea & 0.89 & 0.89 & 0.89 & 0.89 & 1.00 & 1.00 & 1.00 \\
\hline ID & Vegetation class & & & & & & & \\
\hline 23 & Broad-Leaved Forest & 0.92 & 0.89 & 0.91 & 0.97 & 1.00 & 1.00 & 1.01 \\
24 & Coniferous Forest & 0.93 & 0.82 & 1.16 & 0.82 & 1.00 & 1.27 & 1.43 \\
25 & Mixed Forest & 0.92 & 0.86 & 1.04 & 0.90 & 1.00 & 1.14 & 1.22 \\
100 & Broadl. Everg. Forest & 0.92 & 0.89 & 0.91 & 0.97 & 1.00 & 1.00 & 1.01 \\
$101 \mathrm{a}$ & Broadl. Dec. Forest & 0.90 & 0.75 & 0.86 & 1.18 & 1.00 & 1.00 & 1.05 \\
$101 \mathrm{~b}$ & Broadl. Dec. Forest & 0.86 & 0.80 & 0.85 & 0.97 & 1.00 & 1.00 & 1.02 \\
$101 \mathrm{c}$ & Broadl. Dec. Forest & 0.92 & 0.89 & 0.91 & 0.97 & 1.00 & 1.00 & 1.01 \\
$102 \mathrm{a}$ & Needl. Everg. Forest & 0.89 & 0.72 & 1.04 & 0.98 & 1.00 & 1.21 & 1.36 \\
$102 \mathrm{~b}$ & Needl. Everg. Forest & 0.93 & 0.82 & 1.16 & 0.82 & 1.00 & 1.27 & 1.43 \\
$102 \mathrm{c}$ & Needl. Everg. Forest & 1.00 & 1.00 & 1.00 & 1.00 & 1.00 & 1.00 & 1.00 \\
103 & Needl. Decid. Forest & 1.00 & 1.00 & 1.00 & 1.00 & 1.00 & 1.00 & 1.00 \\
$104 \mathrm{a}$ & Mixed Forest & 0.89 & 0.73 & 0.95 & 1.08 & 1.00 & 1.10 & 1.21 \\
$104 \mathrm{~b}$ & Mixed Forest & 0.92 & 0.86 & 1.04 & 0.90 & 1.00 & 1.14 & 1.22 \\
$104 \mathrm{c}$ & Mixed Forest & 0.92 & 0.86 & 1.04 & 0.90 & 1.00 & 1.14 & 1.22 \\
\hline
\end{tabular}

a Bioclimatic factors are derived from litterfall fluxes of major tree species from different databases as described in the text. Litterfall flux measurements were attributed to the respective vegetation zone where the forest site is located in. A bioclimatic correction factor of 1 was used for the following vegetation zones: Anatolian, Black Sea, Pannonian, and Africa-Arabia. No data on the selected European tree species were found for these zones. The following forest types from the CLC/GLC2000 land use database are assigned a bioclimatic correction factor of 1 in all vegetation zones: forest and shrub/grassland mosaic, regularly flooded saline tree area. All tree species not listed here are assigned a bioclimatic correction factor of 1 in all vegetation zones.

$\mathrm{b}$ Forest sample sites with elevation $>900 \mathrm{~m}$ south of $60^{\circ} \mathrm{N}$ and $>500 \mathrm{~m}$ north of $60^{\circ} \mathrm{N}$ are defined as Alpine. In case no data for one of the listed tree species was found in the Mediterranean or Steppic vegetation zone, the reference value of 1.00 is used. In case no data was found for the Boreal zone, the value from the Alpine zone is used. In case no data was found for Arctic or Alpine zones, the value from the Boreal zone is used instead.

${ }^{c}$ Vegetation class ID of CLC/GLC2000 landcover mosaic as indicated in Köble (2007). Letter a refers to GLC Northern Eurasia v4.0, letter b refers to GLC Europe v1.0 and letter c refers to GLC Africa v5.0 + GLC Global v1.1.

the Pan-European domain defined in this work. FAOSTAT is the most widely recognized standard for national level agricultural statistics. National yields (yearly average of 20002005) of crop species given in the Agricultural Land Use $M a p$ were retrieved from FAOSTAT. The national crop yields were allocated to the vegetation zone to which main parts of the respective country belong geographically (see explanations in Table 2). In the Arctic vegetation zone, the bioclimatic correction factor is 0.55 for all crops and agriculture types. This value is derived from the yield of potatoes grown in Iceland. It should be noted that FAO crop yields not only reflect the influence of climate on agriculture productivity but also depend on agriculture management, farming practices and fertiliser application. Zonal bioclimatic correction factors for crop species and agriculture are compiled in Table 2.
The Foliar biomass density $d_{t}$ for several tree species was derived from the four forest biomass databases (DAAC, ICP, global database by Luyssaert et al. (2007), and AFOLU). This was done for tree species with more than 10 data records within the Continental vegetation zone. Based on 34 records a value of $350 \pm 108 \mathrm{~g} \mathrm{~m}^{-2}$ was obtained for Fagus sylvatica which is at the higher end of the range $\left(310 \pm 47 \mathrm{~g} \mathrm{~m}^{-2}\right)$ given by Veldt (1991). For European oaks (Quercus robur and Quercus petraea) a value of $290 \pm 74 \mathrm{~g} \mathrm{~m}^{-2}$ was derived, which is lower than the mean value of $330 \pm 90 \mathrm{~g} \mathrm{~m}^{-2}$ ) from Veldt (1991) but well within the range of uncertainty. Our values for Betula spp., Pinus sylv. and Picea abies, $230 \pm 185 \mathrm{~g} \mathrm{~m}^{-2}, 690 \pm 240 \mathrm{~g} \mathrm{~m}^{-2}$, and $1340 \pm 700 \mathrm{~g} \mathrm{~m}^{-2}$, respectively, are in reasonable agreement with Veldt (1989), but show a large spread. Furthermore, foliar biomass densities could be derived for the Mediterranean oaks Quercus ilex 
Table 2. Bioclimatic correction for crops and agriculture vegetation classes in European vegetation zones. The Continental vegetation zone is used as the reference zone ${ }^{\mathrm{a}}$.

\begin{tabular}{|c|c|c|c|c|c|c|c|c|c|c|}
\hline $\begin{array}{l}\text { Crop species or } \\
\text { Vegetation class }\end{array}$ & $\begin{array}{l}\text { Alpine } \\
\mathrm{h}\end{array}$ & Anatol. & Atlantic & $\begin{array}{l}\text { Black } \\
\text { Sea }\end{array}$ & Boreal & Contin. & Mediterr. & Pannon. & Steppic & $\begin{array}{l}\text { Africa- } \\
\text { Arabia }\end{array}$ \\
\hline Barley & 0.90 & 0.59 & 1.51 & 0.43 & 0.67 & 1.00 & 0.66 & 0.82 & 0.51 & 0.36 \\
\hline Durum Wheat ${ }^{\mathrm{b}}$ & 0.87 & 0.47 & 1.72 & 0.40 & 0.74 & 1.00 & 0.59 & 0.86 & 0.55 & 0.49 \\
\hline Fruit trees and berry plantations $s^{\mathrm{c}}$ & 0.98 & 1.00 & 1.39 & 0.13 & 0.27 & 1.00 & 0.72 & 0.68 & 0.19 & 0.66 \\
\hline Maize & 0.88 & 0.74 & 1.68 & 0.32 & 0.46 & 1.00 & 1.25 & 0.86 & 0.49 & 1.15 \\
\hline Olive groves & 1.70 & 1.45 & 0.74 & $1.42^{\mathrm{d}}$ & $1.00^{\mathrm{d}}$ & 1.00 & 1.42 & $1.00^{\mathrm{d}}$ & $1.00^{\mathrm{d}}$ & 1.40 \\
\hline Rape and turnip rape & 1.02 & 0.22 & 1.46 & $0.66^{\mathrm{d}}$ & 0.72 & 1.00 & 0.66 & 0.83 & 0.46 & 0.62 \\
\hline Fibre and oleaginous crops $\mathrm{e}^{\mathrm{e}}$ & 1.02 & 0.22 & 1.46 & $0.66^{\mathrm{d}}$ & 0.72 & 1.00 & 0.66 & 0.83 & 0.46 & 0.62 \\
\hline Vineyards & 1.00 & 0.96 & 0.71 & 0.42 & 0.72 & 1.00 & 1.13 & 1.05 & 0.65 & 1.28 \\
\hline Oats & 0.92 & 0.62 & 1.78 & 0.37 & 0.77 & 1.00 & 0.52 & 0.72 & 0.49 & 0.23 \\
\hline Other cereals ${ }^{\mathrm{f}}$ & 0.92 & 0.34 & 1.29 & 0.14 & 0.58 & 1.00 & 0.41 & 0.73 & 0.16 & 0.40 \\
\hline Other crops ${ }^{f}$ & 0.92 & 0.34 & 1.29 & 0.14 & 0.58 & 1.00 & 0.41 & 0.73 & 0.16 & 0.40 \\
\hline Fodder other on arable land & 0.58 & 1.09 & 0.77 & $1.17^{\mathrm{d}}$ & 0.31 & 1.00 & 1.17 & $1.00^{\mathrm{d}}$ & $1.00^{\mathrm{d}}$ & 1.34 \\
\hline Other non perman. industrial $\mathrm{crops}^{\mathrm{e}}$ & 0.92 & 0.34 & 1.29 & 0.14 & 0.58 & 1.00 & 0.41 & 0.73 & 0.16 & 0.40 \\
\hline Tomatoes and Fresh Vegetables ${ }^{\mathrm{g}}$ & 0.82 & 0.72 & 0.59 & 0.56 & 0.62 & 1.00 & 0.78 & 0.75 & 0.51 & 0.70 \\
\hline Rice & 1.15 & 1.48 & 1.32 & 0.00 & 0.85 & 1.00 & 1.39 & 0.89 & 0.90 & 1.23 \\
\hline Potatoes & 0.86 & 1.12 & 1.76 & 0.47 & 0.75 & 1.00 & 0.88 & 0.93 & 0.41 & 0.97 \\
\hline Dry pulses & 1.31 & 0.27 & 1.67 & $0.58^{\mathrm{d}}$ & 0.76 & 1.00 & 0.58 & 0.45 & 0.65 & 0.43 \\
\hline Other root crops & 0.86 & 1.12 & 1.76 & 0.47 & 0.75 & 1.00 & 0.88 & 0.93 & 0.41 & 0.97 \\
\hline Rye & 0.96 & 0.50 & 1.27 & 0.26 & 0.76 & 1.00 & 0.54 & 0.63 & 0.50 & 0.43 \\
\hline Soya & 1.38 & 2.24 & 1.75 & 1.87 & 0.75 & 1.00 & 1.68 & 1.42 & 0.88 & 1.03 \\
\hline Sugar beet & 1.02 & 0.96 & 1.39 & 0.38 & 1.22 & 1.00 & 1.36 & 1.02 & 0.48 & 1.10 \\
\hline Sunflower & 0.68 & 0.78 & 1.20 & 0.32 & 0.45 & 1.00 & 0.62 & 1.03 & 0.61 & 0.75 \\
\hline Common wheat ${ }^{\mathrm{b}}$ & 0.87 & 0.47 & 1.72 & 0.40 & 0.74 & 1.00 & 0.59 & 0.86 & 0.55 & 0.49 \\
\hline Non-Irrigated Arable Land ${ }^{\mathrm{b}}$ & 0.87 & 0.47 & 1.72 & 0.40 & 0.74 & 1.00 & 0.59 & 0.86 & 0.55 & 0.49 \\
\hline Permanently Irrigated Land ${ }^{g}$ & 0.82 & 0.72 & 0.59 & 0.56 & 0.62 & 1.00 & 0.78 & 0.75 & 0.51 & 0.70 \\
\hline Rice Fields & 1.15 & 1.48 & 1.32 & $1.39^{\mathrm{d}}$ & 0.85 & 1.00 & 1.39 & 0.89 & 0.90 & 1.23 \\
\hline Vineyards & 1.00 & 0.96 & 0.71 & 0.42 & 0.72 & 1.00 & 1.13 & 1.05 & 0.65 & 1.28 \\
\hline Olive Groves & 1.70 & 1.45 & 0.74 & $1.42^{\mathrm{d}}$ & $1.00^{\mathrm{d}}$ & 1.00 & 1.42 & $1.00^{\mathrm{d}}$ & $1.00^{\mathrm{d}}$ & 1.40 \\
\hline Pastures & 0.58 & 1.09 & 0.77 & $1.17^{\mathrm{d}}$ & 0.31 & 1.00 & 1.17 & $1.00^{\mathrm{d}}$ & $1.00^{\mathrm{d}}$ & 1.34 \\
\hline Annual crops assoc. w perm. crops ${ }^{\mathrm{e}}$ & 0.92 & 0.34 & 1.29 & 0.14 & 0.58 & 1.00 & 0.41 & 0.73 & 0.16 & 0.40 \\
\hline Complex Cultivation Patterns & 1.14 & 0.38 & 1.47 & 0.13 & 0.76 & 1.00 & 0.56 & 0.54 & 0.58 & 0.43 \\
\hline Agriculture w. areas of natural veg. & 0.94 & 0.42 & 1.28 & 0.20 & 0.67 & 1.00 & 0.48 & 0.68 & 0.33 & 0.42 \\
\hline Agro-Forestry Areas & 1.54 & 1.84 & 1.24 & 0.94 & 0.37 & 1.00 & 1.55 & 0.71 & 0.44 & 1.21 \\
\hline Non Irrigated Cropland ${ }^{b}$ & 0.87 & 0.47 & 1.72 & 0.40 & 0.74 & 1.00 & 0.59 & 0.86 & 0.55 & 0.49 \\
\hline Irrigated Cropland ${ }^{\mathrm{g}}$ & 0.82 & 0.72 & 0.59 & 0.56 & 0.62 & 1.00 & 0.78 & 0.75 & 0.51 & 0.70 \\
\hline Cropland unspecified & 1.04 & 0.91 & 1.31 & $0.90^{\mathrm{d}}$ & 0.71 & 1.00 & 0.90 & 0.81 & 0.53 & 0.81 \\
\hline
\end{tabular}

a Bioclimatic factors are derived from FAO crop yields reported by the respective nations that were allocated to a specific vegetation zone. The Anatolian zone is represented by Turkey, the Alpine zone by Bosnia and Herzegovina, Macedonia, Slovakia, Slovenia, Switzerland, the Atlantic zone by Belgium, Denmark, France, Ireland, the Netherlands, United Kingdom, the Black Sea zone by Georgia, the Boreal zone by Estonia, Finland, Latvia, Lithuania, the Russian Federation, Sweden, the Continental zone by Austria, Belarus, Bulgaria, Croatia, France, Germany, Luxembourg, Poland, Romania, the Mediterranean zone by Albania, Greece, Italy, Malta, Portugal, Spain, Cyprus, the Pannonian zone by Hungary, the Steppic zone by Moldavia, Ukraine, the Africa-Arabia zone by Israel, Jordan, Lebanon, Syrian Arab Republic, Algeria, Egypt, Libyan Arab Jamahiriya, Morocco, Tunisia. The following crops and agriculture types are assigned a bioclimatic factor of 1 in all vegetation zones except for the Arctic: citrus fruits, nurseries, floriculture, fallow land, permanent gras and grazing, and tobacco from the Agricultural Land Use Map, and the vegetation classes tree crops and cropland/forest/shrubland/grassland mosaic from CLC/GLC2000. In the Arctic vegetation zone, the bioclimatic factor is 0.55 for all crops and agriculture types.

${ }^{b}$ From FAO crop yields of wheat; ${ }^{c}$ From FAO crop yields of apple; ${ }^{d}$ No yields reported to FAO, the bioclimatic factor from Mediterranean is used for Black Sea and the bioclimatic factor from Continental is used for other zones; ${ }^{\mathrm{e}}$ From FAO crop yields of rape seed; ${ }^{\mathrm{f}}$ From FAO crop yields of cereals; ${ }^{\mathrm{g}}$ From FAO crop yields of vegetables.

$\mathrm{h}$ We note that high values $\left(f_{c}>1\right)$ for olive groves, rice, soya and dry pulses in the Alpine zone are a result of defining certain countries as alpine though only parts of the country belong to the Alpine zone. The high values have no effect on the calculation of the emissions since the mentioned crops are not cultivated in the Alpine zone. 
and Quercus coccifera. They were found to be $510 \mathrm{~g} \mathrm{~m}^{-2}$ and $520 \mathrm{~g} \mathrm{~m}^{-2}$, respectively, and thus well in agreement with the default value for evergreen broadleaved trees given by Veldt (1989).

Foliar biomass density values of trees, $d_{t}$, and of crops, $d_{c}$, for use with Eq. (6) are listed in Table 3 and 4. Most values in Table 3 and 4 are representative for growing conditions in the temperate and humid part of Europe, which is in our work referred to as the Continental zone. To determine $D_{i}$, foliar biomass densities of main tree and all crop species and classes were corrected with the bioclimatic factor for different climatic regions. Foliar biomass densities of trees were adopted from the EMEP/CORINAIR (1999) manual, which are based on the study of Veldt (1989), and for Mediterranean trees from Owen et al. (2001) and completed with values from Geron et al. (1994) taken from the GloBEIS database. For several tree species, foliar biomass densities have been derived from litterfall data within the frame of this work. For crop species, default values for foliar biomass densities given in the EMEP/CORINAIR (1999) manual and from Simpson et al. (1999) were taken. The plant composition of CLC/GLC2000 vegetation classes was extracted from the CLC/GLC2000 documentation. The plant composition (see Table 5) was used to derive foliar biomass densities for the respective CLC/GLC2000 class by weighting the contributions of individual crop species from the Agricultural Land Use Map and tree species from the Tree Species Map. This procedure reduces the bias of foliar biomass densities for general land use classes, compared to the application of default values for general land use classes.

\subsection{BVOC standard emission factors}

The aggregation of the standard emission potential $\epsilon_{b, i}$ of a BVOC class $b$ in a grid cell follows the procedure for the foliar densities; it is:

$\epsilon_{b, i}=\sum_{t} a_{t, i}^{\prime} \epsilon_{b, t, i}+\sum_{c} a_{c, i}^{\prime} \epsilon_{b, c, i}+\sum_{g} a_{g, i} \epsilon_{b, g, i}$

where $\epsilon_{b, t, i}, \epsilon_{b, c, i}$ and $\epsilon_{b, g, i}$ are the BVOC standard emission potentials of a single tree species, crop species and CLC/GLC2000 vegetation class respectively. Data on emission potentials at standard conditions for tree and crops species and for the CLC/GLC2000 classes was adopted from the work of Steinbrecher et al. (2009), the NatAir project inventory. European standard emission potential distributions calculated from Eq. (7) for isoprene, monoterpenes (pool), monoterpenes (synthesis), and sesquiterpenes are shown in Fig. A2 (in the Electronic Supplement: http://www.biogeosciences.net/6/1059/ 2009/bg-6-1059-2009-supplement.pdf). A complete list of standard emission potentials for all BVOC classes of trees, $\epsilon_{b, t, i}$, and of crops, $\epsilon_{b, c, i}$ is given in Tables 3 and 4. Vegetation composition, foliar biomass densities and BVOC standard emission potentials for CLC/GLC2000 vegetation classes are compiled in Table 5.

Monoterpenes are known to constitute a main fraction of "essential oils" that are produced and stored in the plant secretory organs (Kesselmeier and Staudt, 1999). It is now well established that stored monoterpenes and other BVOC are released depending on the temperature and this type of emission behaviour is usually referred to as pool emissions. Isoprene is never stored after its production by plants but rapidly lost by volatilisation and isoprene emissions are dependent on both temperature and light. A number of studies revealed that monoterpene (MT) emissions from many plant species exhibit a strong dependence on light and were negligible during the night (Staudt and Seufert, 1995; Bertin et al., 1997; Moukhtar et al., 2005; Dindorf et al., 2006). This type of emissions is termed newly synthesized emissions.

Crop plants are considered to be low emitting species. However, grasslands and pastures have been found to emit quite substantial amounts of oxygenated VOC (Kesselmeier and Staudt, 1999). Previous estimates of European BVOC emissions from agriculture have been based on generalized crop emission properties (e.g. Simpson et al., 1995, 1999; Steinbrecher et al., 2009). Simpson et al. (1999) estimate a total of $1440 \mathrm{Gg} \mathrm{yr}^{-1}$ BVOC from agriculture crops, consisting mainly of oxygenated VOC emissions (1350 $\mathrm{Gg} \mathrm{yr}^{-1}$ ). Isoprene emissions from widespread crops as wheat, oat and rye are generally found to be zero or close to zero (Arey et al., 1991; Winer et al., 1992; König et al.,1995). Available emission data for oxygenated VOC (OVOC) are scarce and only rough estimations on oxygenated VOC emissions exist (e.g. Seco et al., 2007, and references therein) and there are only a few studies with chemical speciation of OVOCs. The OVOC class includes all oxygenated compounds with $\mathrm{C} 1-\mathrm{C} 12$, like alcohols, ketones, aldehydes, etc., which are volatile. A large fraction of oxygenated VOC (often abbreviated as oxyVOC) is composed of short-chained VOC, like methanol, ethanol, formaldehyde, acetaldehyde, acetone, formic acid, and acetic acid. In this work, the standard emission potentials for OVOC were adopted from the NatAir study (Steinbrecher et al., 2009). For most plant species and land use classes a default value of $2.0 \mu \mathrm{g} \mathrm{g}_{\mathrm{DW}}^{-1} \mathrm{~h}^{-1}$ is applied.

Information on plant-specific emission factors for sesquiterpenes in Europe has been compiled in the recent review of Duhl et al. (2007). Duhl et al. (2007) state that values of early sesquiterpene (SQT) emission studies were very likely positively biased by disturbance-induced emission bursts, elevated enclosure temperatures and disturbances during sample collection. In the Duhl et al. (2007) review, average values of SQT standard emission potentials for coniferous trees, broadleaf trees, shrubs and crops are presented but these authors note that the high standard deviations associated with each class preclude using these data to assign emission factors to individual plant functional types since there is no significant difference between these average values. The respective average and standard 
Table 3. Foliar biomass densities in $\mathrm{g}$ dry mass $\mathrm{m}^{-2}$ and standard emission potentials (in $\mu \mathrm{g} \mathrm{g}_{\mathrm{DW}}^{-1} \mathrm{hr}^{-1}$ at $30^{\circ} \mathrm{C}$ leaf temperature and $1000 \mu \mathrm{mol} \mathrm{m}^{-2} \mathrm{~s}^{-1}$ PPFD) of isoprene, monoterpenes, oxygenated VOC (OVOC) and sesquiterpenes for tree species. $\mathrm{MT}_{\mathrm{synt}}$ are monoterpenes emitted with temperature and light dependence and $\mathrm{MT}_{\text {pool }}$ are only temperature dependent. Standard emission potentials are adopted

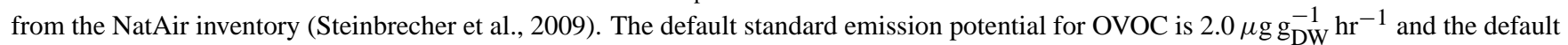
standard emission potential for sesquiterpenes is $0.1 \mu \mathrm{g} \mathrm{g}_{\mathrm{DW}}^{-1} \mathrm{hr}^{-1}$. A. f. is the percentage area of each tree species to the total forest area of the Tree Species Map, taken from Lenz et al. (2002). Seasonality type: eve=broadleaf/coniferous evergreen, dec=broadleaf/coniferous deciduous.

\begin{tabular}{|c|c|c|c|c|c|c|c|c|c|}
\hline \multirow[t]{2}{*}{ Species } & \multirow{2}{*}{$\begin{array}{l}d \\
\left(\mathrm{gm}^{-2}\right)\end{array}$} & \multicolumn{5}{|c|}{ Stand. emission potential $\left(\mu \mathrm{g} \mathrm{g}_{\mathrm{DW}}^{-1} \mathrm{hr}^{-1}\right)$} & \multirow{2}{*}{$\begin{array}{l}\text { Seas. } \\
\text { type }\end{array}$} & \multirow{2}{*}{$\begin{array}{l}\text { A. f. } \\
(\%)\end{array}$} & \multirow[t]{2}{*}{ Ref. $d$} \\
\hline & & ISOP & $\mathrm{MT}_{\text {synt }}$ & $\mathrm{MT}_{\text {pool }}$ & OVOC & SQT & & & \\
\hline Abies alba & 1260 & 1.0 & 0.5 & 1.0 & 2.0 & 0.1 & eve & 1.505 & AAK95 \\
\hline Abies borisii-regis & 1260 & 18.4 & 2.5 & 0.2 & 2.0 & 0.1 & eve & 0.149 & Abies alba \\
\hline Abies cephalonica & 1260 & 0.0 & 0.3 & 0.6 & 2.0 & 0.1 & eve & 0.223 & Abies alba \\
\hline Abies grandis & 1260 & 0.0 & 0.3 & 0.6 & 2.0 & 0.1 & eve & 0.003 & Abies alba \\
\hline Acer campestre & 320 & 0.0 & 1.5 & 0.0 & 2.0 & 0.1 & dec & 0.046 & $\mathrm{CO} 99,{ }^{1}$ \\
\hline Acer monspessulanum & 320 & 0.0 & 1.5 & 0.0 & 2.0 & 0.1 & dec & 0.020 & CO99, ${ }^{1}$ \\
\hline Acer opalus & 320 & 0.1 & 1.5 & 0.0 & 2.0 & 0.1 & dec & 0.044 & $\mathrm{CO} 99,1$ \\
\hline Acer platanoides & 320 & 0.1 & 1.5 & 0.0 & 2.0 & 0.1 & dec & 0.337 & $\mathrm{CO} 99,{ }^{1}$ \\
\hline Acer sp. & 320 & 0.1 & 1.5 & 0.0 & 2.0 & 0.1 & dec & 0.122 & $\mathrm{CO} 99,1$ \\
\hline Alnus cordata & 320 & 0.0 & 1.5 & 0.0 & 2.0 & 0.1 & dec & 0.072 & $\mathrm{CO} 99,{ }^{2}$ \\
\hline Alnus glutinosa & 320 & 0.0 & 1.5 & 0.0 & 2.0 & 0.1 & dec & 0.346 & $\mathrm{CO} 99,{ }^{2}$ \\
\hline Alnus incana & 320 & 0.0 & 1.5 & 0.0 & 2.0 & 0.1 & dec & 0.188 & $\mathrm{CO} 99,^{2}$ \\
\hline Alnus viridis & 320 & 0.0 & 1.5 & 0.0 & 2.0 & 0.1 & dec & 0.004 & $\mathrm{CO} 99,{ }^{2}$ \\
\hline Arbutus andrachne & 300 & 0.0 & 0.0 & 0.0 & 2.0 & 0.1 & dec & 0.009 & VE89, ${ }^{1}$ \\
\hline Arbutus unedo & 300 & 0.1 & 0.0 & 0.1 & 2.0 & 0.1 & dec & 0.042 & VE89, 1 \\
\hline Betula pendula & 240 & 0.0 & 0.0 & 3.0 & 2.0 & 2.0 & dec & 2.321 & This work \\
\hline Betula pubescens & 240 & 0.0 & 0.0 & 3.0 & 2.0 & 2.0 & dec & 4.661 & This work \\
\hline Buxus sempervirens & 980 & 10.0 & 0.0 & 0.2 & 2.0 & 0.1 & eve & 0.025 & OW01 \\
\hline Carpinus betulus & 320 & 0.0 & 0.0 & 0.1 & 2.0 & 0.1 & dec & 0.970 & $\mathrm{CO} 99,^{3}$ \\
\hline Carpinus orientalis & 320 & 0.0 & 0.0 & 0.0 & 2.0 & 0.1 & dec & 0.012 & $\mathrm{CO} 99,{ }^{3}$ \\
\hline Castanea sativa & 380 & 0.0 & 10.0 & 0.0 & 2.0 & 0.1 & dec & 1.077 & VE89 \\
\hline Cedrus atlantica & 700 & 0.0 & 0.0 & 1.0 & 2.0 & 0.1 & eve & 0.024 & $\mathrm{CO} 99,{ }^{4}$ \\
\hline Cedrus deodara & 700 & 0.0 & 0.0 & 1.0 & 2.0 & 0.1 & eve & 0.003 & $\mathrm{CO} 99,{ }^{4}$ \\
\hline Cercis siliquastrum & 300 & 0.0 & 0.0 & 0.0 & 2.0 & 0.1 & dec & 0.011 & VE89, ${ }^{1}$ \\
\hline Ceratonia siliqua & 300 & 0.0 & 0.0 & 0.0 & 2.0 & 0.1 & eve & 0.003 & VE89, ${ }^{1}$ \\
\hline Corylus avellana & 300 & 0.0 & 0.0 & 0.0 & 2.0 & 0.1 & dec & 0.010 & VE89, ${ }^{1}$ \\
\hline Cupressus sempervirens & 700 & 0.0 & 0.0 & 0.7 & 2.0 & 0.1 & eve & 0.030 & CO99 \\
\hline Erica arborea & 96 & 13.0 & 0.0 & 0.0 & 2.0 & 0.1 & eve & 0.011 & OW01 \\
\hline Erica multiflora & 150 & 0.0 & 0.0 & 0.0 & 2.0 & 0.1 & eve & $<0.001$ & OW01 \\
\hline Erica scoparia & 150 & 0.0 & 0.0 & 0.0 & 2.0 & 0.1 & eve & $<0.001$ & OW01 \\
\hline Eucalyptus sp. & 400 & 50.0 & 0.0 & 5.4 & 2.0 & 0.1 & eve & 0.847 & CO99 \\
\hline Fagus moesiaca & 320 & 0.0 & 0.0 & 0.0 & 2.0 & 0.1 & dec & 0.148 & $\mathrm{CO} 99,5$ \\
\hline Fagus orientalis & 320 & 0.0 & 0.0 & 0.0 & 2.0 & 0.1 & dec & 0.015 & $\mathrm{CO} 99,^{5}$ \\
\hline Fagus sylvatica & 350 & 0.0 & 21.1 & 0.0 & 2.0 & 0.1 & dec & 7.107 & This work \\
\hline Fraxinus angustifolia & 320 & 0.0 & 0.0 & 0.0 & 2.0 & 0.1 & dec & 0.049 & $\mathrm{CO} 99,6$ \\
\hline Fraxinus excelsior & 320 & 0.0 & 0.0 & 0.0 & 2.0 & 0.1 & dec & 0.596 & $\mathrm{CO} 99,6$ \\
\hline Fraxinus ornus & 320 & 0.0 & 0.0 & 0.0 & 2.0 & 0.1 & dec & 0.091 & $\mathrm{CO} 99,{ }^{6}$ \\
\hline Ilex aquifolium & 375 & 0.0 & 0.0 & 0.0 & 2.0 & 0.1 & eve & 0.006 & GE94 \\
\hline Juglans nigra & 320 & 0.0 & 0.0 & 1.0 & 2.0 & 0.1 & dec & 0.001 & CO99 \\
\hline Juglans regia & 300 & 0.0 & 0.0 & 1.0 & 2.0 & 0.1 & dec & 0.003 & VE89 \\
\hline Juniperus communis & 700 & 0.0 & 0.3 & 0.6 & 2.0 & 0.1 & eve & 0.025 & $\mathrm{CO} 99,{ }^{7}$ \\
\hline Juniperus oxycedrus & 700 & 0.0 & 0.0 & 1.5 & 2.0 & 0.1 & eve & 0.038 & $\mathrm{CO} 99,{ }^{7}$ \\
\hline Juniperus phoenicea & 700 & 0.0 & 0.0 & 1.5 & 2.0 & 0.1 & eve & 0.015 & CO99, ${ }^{7}$ \\
\hline Juniperus thurifera & 700 & 0.0 & 0.0 & 0.0 & 2.0 & 0.1 & eve & 0.147 & $\mathrm{CO} 99,{ }^{7}$ \\
\hline Larix decidua & 300 & 0.0 & 0.0 & 5.0 & 2.0 & 0.1 & dec & 0.808 & VE89, ${ }^{2}$ \\
\hline
\end{tabular}


Table 3. Continued.

\begin{tabular}{|c|c|c|c|c|c|c|c|c|c|}
\hline \multirow[t]{2}{*}{ Species } & \multirow{2}{*}{$\begin{array}{l}d \\
\left(\mathrm{gm}^{-2}\right)\end{array}$} & \multicolumn{5}{|c|}{ Stand. emission potential $\left(\mu \mathrm{gg}_{\mathrm{DW}}^{-1} \mathrm{hr}^{-1}\right)$} & \multirow{2}{*}{$\begin{array}{l}\text { Seas. } \\
\text { type }\end{array}$} & \multirow{2}{*}{$\begin{array}{l}\text { A. f. } \\
(\%)\end{array}$} & \multirow[t]{2}{*}{ Ref. $d$} \\
\hline & & ISOP & $\mathrm{MT}_{\text {synt }}$ & $\mathrm{MT}_{\text {pool }}$ & OVOC & SQT & & & \\
\hline Larix kaempferi & 300 & 0.0 & 0.0 & 5.0 & 2.0 & 0.1 & dec & 0.065 & VE89, ${ }^{2}$ \\
\hline Laurus nobilis & 500 & 0.0 & 0.0 & 0.0 & 2.0 & 0.1 & eve & $<0.001$ & VE89, ${ }^{3}$ \\
\hline Malus domestica & 375 & 0.0 & 0.0 & 0.0 & 2.0 & 0.1 & dec & 0.002 & GE94 \\
\hline Olea europaea & 200 & 0.0 & 0.0 & 0.1 & 2.0 & 0.1 & eve & 0.041 & CO99 \\
\hline Ostrya carpinifolia & 320 & 0.0 & 0.0 & 0.0 & 2.0 & 0.1 & dec & 0.288 & $\mathrm{CO} 99,^{3}$ \\
\hline Phillyrea latifolia & 300 & 0.0 & 0.0 & 0.5 & 2.0 & 0.1 & eve & 0.028 & VE89, ${ }^{1}$ \\
\hline Picea abies & 1340 & 1.0 & 2.1 & 0.4 & 2.0 & 0.1 & eve & 21.36 & This work \\
\hline Picea sitchensis & 1400 & 4.8 & 0.0 & 6.5 & 2.0 & 0.1 & eve & 0.776 & VE89 \\
\hline Pinus brutia & 700 & 0.0 & 0.0 & 2.0 & 2.0 & 0.1 & eve & 0.040 & VE89, ${ }^{4}$ \\
\hline Pinus canariensis & 700 & 0.0 & 0.0 & 6.0 & 2.0 & 0.1 & eve & 0.062 & VE89, ${ }^{4}$ \\
\hline Pinus cembra & 700 & 0.0 & 0.0 & 2.5 & 2.0 & 0.1 & eve & 0.020 & VE89, ${ }^{4}$ \\
\hline Pinus contorta & 700 & 0.0 & 0.0 & 6.0 & 2.0 & 0.1 & eve & 0.198 & VE89, ${ }^{4}$ \\
\hline Pinus halepensis & 700 & 0.0 & 0.0 & 2.7 & 2.0 & 0.1 & eve & 1.296 & VE89, ${ }^{4}$ \\
\hline Pinus leucodermis & 700 & 0.0 & 0.0 & 6.0 & 2.0 & 0.1 & eve & 0.008 & VE89, ${ }^{4}$ \\
\hline Pinus mugo & 700 & 0.0 & 0.0 & 6.0 & 2.0 & 0.1 & eve & 0.002 & VE89, ${ }^{4}$ \\
\hline Pinus nigra & 700 & 0.0 & 3.0 & 3.0 & 2.0 & 0.1 & eve & 1.735 & VE89, ${ }^{4}$ \\
\hline Pinus pinaster & 700 & 0.0 & 0.0 & 2.0 & 2.0 & 0.1 & eve & 2.532 & VE89, ${ }^{4}$ \\
\hline Pinus pinea & 700 & 0.0 & 3.0 & 3.0 & 1.8 & 0.1 & eve & 0.233 & VE89, 4 \\
\hline Pinus radiata & 700 & 0.0 & 3.0 & 3.0 & 2.0 & 0.1 & eve & 0.136 & VE89, ${ }^{4}$ \\
\hline Pinus strobus & 700 & 0.0 & 2.5 & 2.5 & 2.0 & 0.1 & eve & 0.031 & VE89, ${ }^{4}$ \\
\hline Pinus sylvestris & 690 & 0.1 & 2.5 & 2.5 & 2.0 & 0.1 & eve & 31.15 & This work \\
\hline Pinus uncinata & 700 & 0.1 & 2.5 & 2.5 & 2.0 & 0.1 & eve & 0.056 & VE89, ${ }^{4}$ \\
\hline Pistacia lentiscus & 1014 & 0.0 & 0.0 & 0.6 & 2.0 & 0.1 & eve & 0.001 & OW01 \\
\hline Pistacia terebinthus & 230 & 0.0 & 0.0 & 0.1 & 2.0 & 0.1 & eve & 0.014 & OW01 \\
\hline Platanus orientalis & 320 & 18.5 & 0.0 & 0.1 & 2.0 & 0.1 & dec & 0.068 & CO99 \\
\hline Populus alba & 320 & 60.0 & 0.0 & 0.0 & 2.0 & 0.1 & dec & 0.031 & $\mathrm{CO} 99,{ }^{8}$ \\
\hline Populus canescens & 320 & 70.0 & 0.0 & 0.0 & 3.5 & 0.1 & dec & 0.060 & $\mathrm{CO} 99,{ }^{8}$ \\
\hline Populus hybrides & 320 & 70.0 & 0.0 & 0.0 & 2.0 & 0.1 & dec & 0.179 & $\mathrm{CO} 99,8$ \\
\hline Populus nigra & 320 & 70.0 & 0.0 & 0.0 & 2.0 & 0.1 & dec & 0.044 & $\mathrm{CO} 99,{ }^{8}$ \\
\hline Populus tremula & 320 & 60.0 & 0.0 & 0.0 & 2.0 & 0.1 & dec & 0.598 & $\mathrm{CO} 99,{ }^{8}$ \\
\hline Prunus avium & 300 & 0.0 & 0.0 & 0.1 & 2.0 & 0.1 & dec & 0.151 & $\mathrm{CO} 99,{ }^{9}$ \\
\hline Prunus padus & 300 & 0.0 & 0.0 & 0.1 & 2.0 & 0.1 & dec & 0.001 & CO99, ${ }^{9}$ \\
\hline Prunus serotina & 300 & 0.0 & 0.0 & 0.1 & 2.0 & 0.1 & dec & 0.005 & $\mathrm{CO} 99,{ }^{9}$ \\
\hline Pseudotsuga menziesii & 1000 & 1.0 & 0.0 & 2.0 & 2.0 & 0.1 & eve & 0.420 & CO99 \\
\hline Pyrus communis & 300 & 0.0 & 0.0 & 0.0 & 2.0 & 0.1 & dec & 0.005 & VE89, ${ }^{1}$ \\
\hline Quercus cerris & 320 & 0.1 & 0.0 & 0.6 & 2.0 & 0.1 & dec & 1.037 & VE89,,$^{5}$ \\
\hline Quercus coccifera & 520 & 0.1 & 25.0 & 0.0 & 2.0 & 0.1 & eve & 0.225 & This work \\
\hline Quercus faginea & 320 & 111.0 & 0.0 & 0.0 & 2.0 & 0.1 & dec & 0.228 & VE89, ${ }^{5}$ \\
\hline Quercus frainetto & 320 & 85.0 & 0.0 & 0.0 & 2.0 & 0.1 & dec & 0.517 & VE89, 5 \\
\hline Quercus fructosa & 320 & 0.1 & 20.0 & 0.0 & 2.0 & 0.1 & eve & 0.048 & VE89, ${ }^{5}$ \\
\hline Quercus ilex & 510 & 0.1 & 43.0 & 0.0 & 2.0 & 0.1 & eve & 2.177 & This work \\
\hline Quercus macrolepsis & 320 & 0.2 & 0.0 & 0.7 & 2.0 & 0.1 & dec & 0.019 & VE89, ${ }^{5}$ \\
\hline Quercus petraea & 290 & 45.0 & 0.0 & 0.3 & 2.0 & 0.1 & dec & 2.309 & This work \\
\hline Quercus pubescens & 320 & 70.0 & 0.0 & 0.3 & 2.0 & 0.1 & dec & 1.488 & VE89, ${ }^{5}$ \\
\hline Quercus pyrenaica & 320 & 59.0 & 0.0 & 0.3 & 2.0 & 0.1 & dec & 0.618 & VE89, ${ }^{5}$ \\
\hline Quercus robur & 290 & 70.0 & 0.0 & 1.0 & 2.0 & 0.1 & dec & 2.930 & This work \\
\hline Quercus rotundifolia & 500 & 0.2 & 14.6 & 0.0 & 2.0 & 0.1 & eve & 0.201 & VE89, ${ }^{3}$ \\
\hline Quercus rubra & 320 & 35.0 & 0.0 & 0.1 & 2.0 & 0.1 & dec & 0.132 & VE89, 5 \\
\hline Quercus suber & 500 & 0.2 & 20.0 & 0.0 & 2.0 & 0.1 & eve & 0.835 & VE89, ${ }^{3}$ \\
\hline Quercus trojana & 320 & 0.2 & 0.0 & 0.2 & 2.0 & 0.1 & eve & 0.041 & $\mathrm{VE} 89,^{5}$ \\
\hline
\end{tabular}


Table 3. Continued.

\begin{tabular}{|c|c|c|c|c|c|c|c|c|c|}
\hline \multirow[t]{2}{*}{ Species } & \multirow{2}{*}{$\begin{array}{l}d \\
\left(\mathrm{gm}^{-2}\right)\end{array}$} & \multicolumn{5}{|c|}{ Stand. emission potential $\left(\mu \mathrm{gg}_{\mathrm{DW}}^{-1} \mathrm{hr}^{-1}\right)$} & \multirow{2}{*}{$\begin{array}{l}\text { Seas. } \\
\text { type }\end{array}$} & \multirow{2}{*}{$\begin{array}{l}\text { A. f. } \\
(\%)\end{array}$} & \multirow[t]{2}{*}{ Ref. $d$} \\
\hline & & ISOP & $\mathrm{MT}_{\text {synt }}$ & $\mathrm{MT}_{\text {pool }}$ & OVOC & SQT & & & \\
\hline Robinia pseudoacacia & 320 & 12.0 & 0.0 & 0.1 & 2.0 & 0.1 & dec & 0.464 & CO99 \\
\hline Salix alba & 150 & 37.2 & 0.0 & 1.1 & 2.0 & 0.1 & dec & 0.003 & CO99, ${ }^{10}$ \\
\hline Salix caprea & 150 & 18.9 & 0.0 & 0.1 & 2.0 & 0.1 & dec & 0.037 & CO99, ${ }^{10}$ \\
\hline Salix cinerea & 150 & 28.0 & 0.0 & 0.8 & 2.0 & 0.1 & dec & 0.001 & CO99, ${ }^{10}$ \\
\hline Salix eleagnos & 150 & 28.0 & 0.0 & 0.8 & 2.0 & 0.1 & dec & 0.001 & CO99, ${ }^{10}$ \\
\hline Salix sp. & 150 & 28.0 & 0.0 & 0.8 & 2.0 & 0.1 & dec & 0.024 & CO99, ${ }^{10}$ \\
\hline Sorbus aria & 300 & 0.0 & 0.0 & 0.0 & 2.0 & 0.1 & dec & 0.036 & VE89, ${ }^{1}$ \\
\hline Sorbus aucuparia & 300 & 0.0 & 0.0 & 0.0 & 2.0 & 0.1 & dec & 0.046 & VE89, ${ }^{1}$ \\
\hline Sorbus domestica & 300 & 0.0 & 0.0 & 0.0 & 2.0 & 0.1 & dec & 0.010 & VE89, ${ }^{1}$ \\
\hline Sorbus torminalis & 300 & 0.0 & 0.0 & 0.0 & 2.0 & 0.1 & dec & 0.016 & VE89, ${ }^{1}$ \\
\hline Thuya sp. & 1500 & 0.0 & 0.0 & 0.6 & 2.0 & 0.1 & eve & 0.003 & GE94 \\
\hline Tilia cordata & 320 & 0.0 & 0.0 & 0.0 & 2.0 & 0.1 & dec & 0.211 & CO99, ${ }^{11}$ \\
\hline Tilia platyphyllos & 320 & 0.0 & 0.0 & 0.0 & 2.0 & 0.1 & dec & 0.044 & CO99, ${ }^{11}$ \\
\hline Tsuga sp. & 700 & 0.1 & 0.0 & 0.2 & 2.0 & 0.1 & eve & 0.020 & GE94 \\
\hline Ulmus glabra & 320 & 0.1 & 0.0 & 0.1 & 2.0 & 0.1 & dec & 0.030 & $\mathrm{CO} 99,{ }^{12}$ \\
\hline Ulmus laevis & 320 & 0.1 & 0.0 & 0.1 & 2.0 & 0.1 & dec & 0.006 & $\mathrm{CO} 99,{ }^{12}$ \\
\hline Ulmus minor & 320 & 0.1 & 0.0 & 0.1 & 2.0 & 0.1 & dec & 0.012 & $\mathrm{CO} 99,{ }^{12}$ \\
\hline
\end{tabular}

References: AAK95 Andreani-Aksoyoglu and Keller (1995); VE89 Veldt (1989), value for: ${ }^{1}$ default deciduous broadleaved, ${ }^{2}$ Larix default, 3 default evergreen broadleaved, ${ }^{4}$ other Pinus spp., ${ }^{5}$ deciduous oaks; CO99 EMEP/CORINAIR (1999), value for ${ }^{1}$ Acer $s p .,{ }^{2}$ Alnus sp., 3 Carpinus, ${ }^{4}$ Cedrus, ${ }^{5}$ Fagus sp.,${ }^{6}$ Fraxinus, ${ }^{7}$ Juniperus, ${ }^{8}$ Populus, ${ }^{9}$ Prunus, ${ }^{10}$ Salix, ${ }^{11}$ Tilia, ${ }^{12}$ Ulmus ; OW01 Owen et al. (2001); GE94 Geron et al. (1994).

deviation values are $0.29 \pm 0.41,1.41 \pm 2.20,7.06 \pm 6.83$ and $0.19 \pm 0.43 \mu \mathrm{g} \mathrm{g}_{\mathrm{DW}}^{-1} \mathrm{~h}^{-1}$. The high standard deviations associated with these numbers allow for the lower default value of $0.1 \mu \mathrm{g} \mathrm{g}_{\mathrm{DW}}^{-1} \mathrm{~h}^{-1}$ that was chosen in this work. We applied the default standard emission potential for all tree species, with the exception of birch. For Betula pendula and Betula pubescens experimental data showed elevated emission potentials (König et al., 1995; Hakola et al., 2001; Vuorinen et al., 2005).

\subsection{Canopy environment model}

In this section a simplified canopy environment model is presented that calculates the emission activity factor $\gamma_{\mathrm{CE}, i}$ in Eq. (1) depending on temperature and the light incident on the leaves as well as on the actual emitting leaf surface (part 4 in Fig. 1). The model contains emission algorithms for different BVOC classes. Depending on compound and plant, emissions originate from the storage pools of the leaves and/or are newly synthesized in the leaves. For pool emissions, the model calculates the pool emission activity factor $\gamma_{\mathrm{CE}, i}$ (pool). For newly synthesized emissions, i.e. emissions of isoprene and $\mathrm{MT}_{\text {synt }}, \gamma_{\mathrm{CE}, i}$ (synt) is calculated.

\subsubsection{Temperature-dependent emissions}

Monoterpene emissions for most coniferous plants and the emissions of OVOC in general are assumed to be pool emissions depending only on temperature. For this compound group, the emission activity factor is:

$\gamma_{\mathrm{CE}, i}(\mathrm{pool})=\gamma_{\mathrm{pool}} \cdot \gamma_{\mathrm{seas}}$

with $\gamma_{\text {pool }}$ calculated using the approach of Guenther et al. (1993):

$\gamma_{\text {pool }}=\exp \left(\beta\left(T-T_{S}\right)\right)$

with the empirical coefficient $\beta=0.09 \mathrm{~K}^{-1}, T$ the leaf temperature (in $\mathrm{K}$ ) and $T_{S}$ the leaf temperature at standard conditions (in $\mathrm{K}$ ). The calculation of $\gamma_{\text {seas }}$ is explained in Sect. 2.4.3.

Emissions of sesquiterpene are assumed to be pool emissions, although there is evidence that emissions of $\beta$ caryophyllene, a major SQT emitted by plants, are both light and temperature dependent (e.g. Schuh et al., 1997; Hansen and Seufert, 2003). Hakola et al. (2006) stated that it can be difficult to discern light dependencies from SQT emission data under field conditions since light saturation of the applied emission algorithms is achieved in most cases. Tarvainen et al. (2005) reported measurements of $\beta$ caryophyllene emissions from Scots Pine in darkened branch 
Table 4. Foliar biomass densities in $\mathrm{g}$ dry mass $\mathrm{m}^{-2}$ and standard emission potentials (in $\mu \mathrm{g} \mathrm{g}_{\mathrm{DW}}^{-1} \mathrm{hr}^{-1}$ at $30^{\circ} \mathrm{C}$ leaf temperature and $1000 \mu \mathrm{mol} \mathrm{m}^{-2} \mathrm{~s}^{-1}$ PPFD) of isoprene, monoterpenes, OVOC and sesquiterpenes for crop species. MT synt are monoterpenes emitted with temperature and light dependence and MT pool are only temperature dependent. Standard emission potentials are adopted from the NatAir inventory (Steinbrecher et al., 2009). The default standard emission potential for OVOC is $2.0 \mu \mathrm{g} \mathrm{g}_{\mathrm{DW}}^{-1} \mathrm{hr}^{-1}$ and the default standard emission potential for sesquiterpenes is $0.1 \mu \mathrm{gg}_{\mathrm{DW}}^{-1} \mathrm{hr}^{-1}$.

\begin{tabular}{|c|c|c|c|c|c|c|c|c|}
\hline \multirow[t]{2}{*}{ Species } & \multirow{2}{*}{$\begin{array}{l}d \\
\left(\mathrm{~g} \mathrm{~m}^{-2}\right)\end{array}$} & \multicolumn{5}{|c|}{ Stand. emission potential $\left(\mu \mathrm{gg}_{\mathrm{DW}}^{-1} \mathrm{hr}^{-1}\right)$} & \multirow{2}{*}{$\begin{array}{l}\text { Seas. }{ }^{a} \\
\text { type }\end{array}$} & \multirow[t]{2}{*}{ Ref. $d$} \\
\hline & & ISOP & $\mathrm{MT}_{\text {synt }}$ & $\mathrm{MT}_{\text {pool }}$ & OVOC & SQT & & \\
\hline Barley & 1290 & 0.0 & 0.5 & 0.5 & 2.0 & 0.1 & barl & CO99 \\
\hline Citrus fruits & 500 & 0.0 & 0.5 & 0.5 & 2.0 & 0.1 & eve & $\mathrm{CO} 99 \mathrm{~b}$ \\
\hline Durum Wheat & 800 & 0.5 & 0.5 & 0.5 & 2.0 & 0.1 & swhe & CO99 \\
\hline Floriculture & 1000 & 0.0 & 0.5 & 0.5 & 2.0 & 0.1 & non & SI99c \\
\hline Permanent gras and grazing & 400 & 0.5 & 0.5 & 0.5 & 2.0 & 0.1 & eve & CO99d \\
\hline Fallow land & 400 & 0.5 & 0.5 & 0.5 & 2.0 & 0.1 & eve & CO99 ${ }^{d}$ \\
\hline Fruit tree and berry plantations & 251 & 0.5 & 0.5 & 0.5 & 2.0 & 0.1 & dec & e \\
\hline Maize & 1610 & 0.0 & 0.5 & 0.5 & 2.0 & 0.1 & maiz & CO99 \\
\hline Olive groves & 200 & 0.0 & 0.5 & 0.5 & 2.0 & 0.1 & eve & CO99 \\
\hline Rape and turnip rape & 400 & 0.0 & 0.5 & 0.5 & 2.0 & 0.1 & rape & CO99 \\
\hline Fibre and oleaginous crops & 400 & 0.0 & 0.5 & 0.5 & 2.0 & 0.1 & sunf & CO99 \\
\hline Vineyards & 410 & 0.0 & 0.5 & 0.5 & 2.0 & 0.1 & maiz & $\mathrm{CO} 99$ \\
\hline Nurseries & 660 & 0.5 & 0.5 & 0.5 & 2.0 & 0.1 & non & f \\
\hline Oats & 750 & 0.5 & 0.5 & 0.5 & 2.0 & 0.1 & barl & CO99 \\
\hline Other cereals & 1335 & 0.5 & 0.5 & 0.5 & 2.0 & 0.1 & swhe & $\mathrm{CO} 99^{\mathrm{g}}$ \\
\hline Other crops & 1335 & 0.5 & 0.5 & 0.5 & 2.0 & 0.1 & swhe & $\mathrm{CO} 99 \mathrm{~g}$ \\
\hline Fodder other on arable land & 400 & 0.5 & 0.5 & 0.5 & 2.0 & 0.1 & maiz & CO99 ${ }^{d}$ \\
\hline Non permanent industrial crops & 1335 & 0.5 & 0.5 & 0.5 & 2.0 & 0.1 & sunf & $\mathrm{CO} 99^{\mathrm{g}}$ \\
\hline Tomatoes and other vegetables & 1000 & 0.0 & 0.5 & 0.5 & 2.0 & 0.1 & non & SI99 ${ }^{c}$ \\
\hline Rice & 1050 & 0.0 & 0.5 & 0.5 & 2.0 & 0.1 & maiz & CO99 \\
\hline Potatoes & 1000 & 0.0 & 0.5 & 0.5 & 2.0 & 0.1 & pota & SI99c \\
\hline Dry pulses & 1000 & 0.0 & 0.5 & 0.5 & 2.0 & 0.1 & rape & SI99c \\
\hline Other root crops & 1000 & 0.0 & 0.5 & 0.5 & 2.0 & 0.1 & roof & SI99 \\
\hline Rye & 400 & 0.0 & 0.5 & 0.5 & 2.0 & 0.1 & swhe & CO99 \\
\hline Soya & 740 & 0.0 & 0.5 & 0.5 & 2.0 & 0.1 & sunf & CO99 \\
\hline Sugar beet & 1000 & 0.0 & 0.5 & 0.5 & 2.0 & 0.1 & roof & SI99c \\
\hline Sunflower & 1000 & 0.0 & 0.5 & 0.5 & 2.0 & 0.1 & sunf & SI99c \\
\hline Common wheat & 800 & 0.5 & 0.5 & 0.5 & 2.0 & 0.1 & swhe & CO99 \\
\hline Tobacco & 490 & 0.0 & 0.5 & 0.5 & 2.0 & 0.1 & sunf & CO99 \\
\hline
\end{tabular}

a Seasonality types: eve=broadleaf/coniferous evergreen, dec=broadleaf/coniferous deciduous, non=no seasonality, barl=spring and winter barley, maiz=fodder and grain maize, swhe=spring and winter wheat, pota=potato, roof=sugar beet, sunf=sunflower, rape=spring and winter rape.

$\mathrm{b}$ Evergreen trees default.

c Crops default.

${ }^{\mathrm{d}}$ Grassland default.

e Mean of Arbutus unedo, Vaccinum, Malus domestica, Pyrus communis.

$\mathrm{f}$ same as Mixed Forest class of CLC2000.

g Miscellaneous crops default.

References: CO99 EMEP/CORINAIR (1999); SI99 Simpson et al. (1999).

cuvettes that were similarly high as when the cuvette received light. For this study the empirical coefficient $\beta$ in Eq. (9) was chosen to be $0.17 \mathrm{~K}^{-1}$ for SQT emissions. This is the average value for $\beta$ given by Helmig et al. (2007) for SQT emissions from eight pine tree species.

\subsubsection{Temperature- and light-dependent emissions}

Isoprene emissions are generally regarded as dependent on light and temperature. Monoterpenes can be released in the same manner as isoprene from many plants (Kesselmeier and Staudt, 1999), a prominent example being European beech 
Table 5. Vegetation composition, Standard emission potential and foliar biomass densities for each CLC and GLC vegetation class ${ }^{\mathrm{a}}$. Foliar biomass density values for agriculture CLC classes are derived from individual crop species as specified in the plant composition list, and taken from Table 4. Standard emission potentials are adopted from the NatAir inventory (Steinbrecher et al., 2009). The plant composition of most GLC vegetation class was extracted from the CLC/GLC2000 documentation or adopted from the corresponding CLC vegetation class. For some GLC classes (e.g. Broadleaved Deciduous Forest in Northern Europe) the plant composition is derived from regions where more details on the vegetation composition were available.

\begin{tabular}{|c|c|c|c|c|c|c|c|c|c|}
\hline \multirow[t]{2}{*}{ ID } & \multirow{2}{*}{$\begin{array}{l}\text { CLC/GLC2000 } \\
\text { Vegetation class }\end{array}$} & \multirow{2}{*}{$\begin{array}{l}d \\
\left(\mathrm{~g} \mathrm{~m}^{-2}\right)\end{array}$} & \multicolumn{5}{|c|}{ Stand. emission potential $\left(\mu \mathrm{gg}_{\mathrm{DW}}^{-1} \mathrm{hr}^{-1}\right)$} & \multirow{2}{*}{$\begin{array}{l}\text { Seas. } \\
\text { type }\end{array}$} & \multirow[t]{2}{*}{ Plant Composition $^{\mathrm{c}}$} \\
\hline & & & ISOP & $\mathrm{MT}_{\text {synt }}$ & $\mathrm{MT}_{\text {pool }}$ & OVOC & SQT & & \\
\hline 10 & Green Urban Areas & 400 & 2.0 & 1.0 & 1.0 & 2.0 & 0.1 & dec & grassland \\
\hline 12 & Non-Irrigated Arable Land & 800 & 0.5 & 0.5 & 0.5 & 2.0 & 0.1 & barl & Durum Wheat \\
\hline 13 & Permanently Irrigated Land & 1000 & 0.5 & 0.5 & 0.5 & 2.0 & 0.1 & maiz & $50 \%$ Tomatoes, $50 \%$ Sugar beet \\
\hline 14 & Rice fields & 1050 & 0.0 & 0.5 & 0.1 & 2.0 & 0.1 & maiz & Rice \\
\hline 15 & Vineyards & 410 & 0.0 & 0.5 & 0.1 & 2.0 & 0.1 & maiz & Vineyards \\
\hline 16 & Fruit trees and berry plantations & 250 & 0.5 & 0.5 & 0.5 & 2.0 & 0.1 & dec & Fruit tree and berry plantations \\
\hline 17 & Olive groves & 200 & 0.0 & 0.5 & 0.5 & 2.0 & 0.1 & eve & Olive groves \\
\hline 18 & Pastures & 400 & 0.5 & 0.5 & 0.5 & 2.0 & 0.1 & dec & Fodder other on arable land \\
\hline 19 & Annual Crops w. perm. crops & 1340 & 0.5 & 0.5 & 0.5 & 2.0 & 0.1 & dec & Non permanent industrial crops \\
\hline 20 & Complex Cultivation Patterns & 330 & 0.5 & 0.5 & 0.5 & 2.0 & 0.1 & dec & $50 \%$ Dry Pulses, 50\% Rye \\
\hline 21 & Agriculture, with natural Veg. & 600 & 0.5 & 0.5 & 0.5 & 2.0 & 0.1 & dec & $50 \%$ Non perm. ind. crops, $50 \%$ Rye \\
\hline 22 & Agro-Forestry Areas & 840 & 1.0 & 1.0 & 1.0 & 2.0 & 0.1 & dec & 25\% Citrus, 25\% Olive, 50\% LC 19 \\
\hline 23 & Broad-Leaved Forest & 360 & 15.0 & 5.0 & 0.5 & 2.0 & 0.1 & dec & d \\
\hline 24 & Coniferous Forest & 950 & 3.0 & 2.5 & 2.5 & 2.0 & 0.1 & eve & $\mathrm{e}$ \\
\hline 25 & Mixed Forest & 660 & 10.0 & 2.0 & 2.0 & 2.0 & 0.1 & $\operatorname{mix}$ & $50 \%$ LC $23,50 \%$ LC 24 \\
\hline 26 & Natural Grassland & 420 & 0.5 & 0.5 & 0.5 & 2.0 & 0.1 & eve & grassland \\
\hline 27 & Moors and Heathland & 350 & 10.0 & 1.0 & 1.0 & 2.0 & 0.1 & eve & moorland/heathland \\
\hline 28 & Sclerophyllous Vegetation & 340 & 5.0 & 15.0 & 15.0 & 2.0 & 0.1 & eve & f \\
\hline 29 & Transitional Woodland-Shrub & 400 & 5.0 & 5.0 & 5.0 & 2.0 & 0.1 & eve & $\mathrm{g}$ \\
\hline 32 & Sparsely Vegetated Areas & 140 & 0.5 & 0.5 & 0.5 & 2.0 & 0.1 & eve & $30 \%$ LC $10,50 \%$ no veg. \\
\hline 35 & Inland Marshes & 200 & 10.0 & 1.0 & 1.0 & 2.0 & 0.1 & eve & marshes \\
\hline 36 & Peatbogs & 330 & 10.0 & 1.0 & 1.0 & 2.0 & 0.1 & eve & ${ }^{h}, 50 \%$ cover \\
\hline 37 & Salt-Marshes & 200 & 10.0 & 1.0 & 1.0 & 2.0 & 0.1 & eve & marshes \\
\hline 100 & Broadleaved Evergreen Forest & 360 & 10.0 & 10.0 & 0.5 & 2.0 & 0.1 & eve & as LC 23 \\
\hline $101 \mathrm{a}$ & Broadleaved Deciduous Forest & 280 & 30.0 & 0.5 & 0.5 & 2.0 & 0.1 & dec & $\mathrm{i}$ \\
\hline $101 \mathrm{~b}$ & Broadleaved Deciduous Forest & 340 & 30.0 & 0.5 & 0.5 & 2.0 & 0.5 & dec & $\mathrm{j}$ \\
\hline $101 \mathrm{c}$ & Broadleaved Deciduous Forest & 250 & 30.0 & 0.5 & 0.5 & 2.0 & 0.1 & dec & as LC $23,70 \%$ cover \\
\hline $102 \mathrm{a}$ & Needleleaved Evergreen Forest & 650 & 3.0 & 2.5 & 2.5 & 2.0 & 0.1 & eve & k, $80 \%$ cover \\
\hline $102 b$ & Needleleaved Evergreen Forest & 950 & 1.0 & 2.5 & 2.5 & 2.0 & 0.1 & eve & as LC 24 \\
\hline $102 \mathrm{c}$ & Needleleaved Evergreen Forest & 700 & 1.0 & 2.5 & 2.5 & 2.0 & 0.1 & eve & l \\
\hline 103 & Needleleaved Deciduous Forest & 300 & 0.5 & 0.0 & 8.0 & 2.0 & 0.1 & dec & Larix decidua \\
\hline $104 \mathrm{a}$ & Mixed Forest & 460 & 10.0 & 3.0 & 3.0 & 2.0 & 0.1 & $\operatorname{mix}$ & $50 \%$ LC $101 \mathrm{a}, 50 \%$ LC $102 \mathrm{a}$ \\
\hline $104 \mathrm{~b}$ & Mixed Forest & 660 & 7.0 & 5.0 & 3.0 & 2.0 & 0.1 & $\operatorname{mix}$ & as LC 25 \\
\hline $104 \mathrm{c}$ & Mixed Forest & 530 & 7.0 & 5.0 & 3.0 & 2.0 & 0.1 & $\operatorname{mix}$ & as LC $25,80 \%$ cover \\
\hline $105 \mathrm{a}$ & Forest, Shrub/Grassland Mos. & 100 & 10.0 & 1.0 & 1.0 & 2.0 & 0.1 & $\operatorname{mix}$ & as $\mathrm{LC} 27,30 \%$ cover \\
\hline $105 \mathrm{~b}$ & Forest, Shrub/Grassland Mos. & 410 & 5.0 & 1.0 & 1.0 & 2.0 & 0.1 & $\operatorname{mix}$ & $70 \%$ LC $29,30 \%$ LC 26 \\
\hline $105 \mathrm{c}$ & Forest, Shrub/Grassland Mos. & 410 & 5.0 & 1.0 & 1.0 & 2.0 & 0.1 & $\operatorname{mix}$ & $70 \%$ LC $29,30 \%$ LC 26 \\
\hline 107 & Evergreen Shrubland & 370 & 3.0 & 2.5 & 2.5 & 2.0 & 0.1 & eve & $50 \%$ LC $28,50 \%$ LC 29 \\
\hline $108 \mathrm{a}$ & Deciduous Shrubland & 330 & 3.0 & 2.5 & 2.5 & 2.0 & 0.1 & dec & $50 \%$ LC 101b, 50\% LC 107 \\
\hline $108 \mathrm{~b}$ & Deciduous Shrubland & 230 & 5.0 & 1.0 & 1.0 & 2.0 & 0.1 & dec & $\mathrm{m}$ \\
\hline $108 \mathrm{c}$ & Deciduous Shrubland & 260 & 5.0 & 1.0 & 1.0 & 2.0 & 0.1 & dec & $\mathrm{n}$ \\
\hline 109 & Grassland & 210 & 0.5 & 0.5 & 0.5 & 2.0 & 0.1 & eve & $50 \%$ LC $26+50 \%$ LC 36 \\
\hline $110 \mathrm{a}$ & Sparse Grassland & 100 & 0.5 & 0.5 & 0.5 & 2.0 & 0.1 & eve & LC $109,50 \%$ cover \\
\hline $110 \mathrm{~b}$ & Sparse Grassland & 210 & 0.5 & 0.5 & 0.5 & 2.0 & 0.1 & eve & LC 109 \\
\hline $110 \mathrm{c}$ & Sparse Grassland & 10 & 0.5 & 0.5 & 0.5 & 2.0 & 0.1 & eve & LC $109,5 \%$ cover \\
\hline $111 \mathrm{a}$ & Wetland unspecified & 330 & 10.0 & 1.0 & 1.0 & 2.0 & 0.1 & eve & LC 36 \\
\hline $111 \mathrm{~b}$ & Wetland unspecified & 330 & 10.0 & 0.5 & 0.5 & 2.0 & 0.1 & eve & LC 36 \\
\hline $111 \mathrm{c}$ & Wetland unspecified & 380 & 10.0 & 0.5 & 0.5 & 2.0 & 0.1 & eve & $50 \%$ LC $36,50 \%$ LC 26 \\
\hline $112 \mathrm{a}$ & Non Irrigated Cropland & 800 & 0.5 & 0.5 & 0.5 & 2.0 & 0.1 & dec & LC 12 \\
\hline $112 \mathrm{~b}$ & Non Irrigated Cropland & 600 & 0.5 & 0.5 & 0.5 & 2.0 & 0.1 & dec & $50 \%$ LC $12,50 \%$ LC 18 \\
\hline $112 \mathrm{c}$ & Non Irrigated Cropland & 520 & 0.5 & 0.5 & 0.5 & 2.0 & 0.1 & dec & $30 \%$ LC $12,70 \%$ LC 18 \\
\hline 113 & Irrigated Cropland & 1000 & 0.5 & 0.5 & 0.5 & 1.0 & 0.1 & dec & LC 13 \\
\hline 114 & Tree Crops & 200 & 0.0 & 0.0 & 0.0 & 2.0 & 0.1 & eve & Date Palm \\
\hline
\end{tabular}


Table 5. Continued.

\begin{tabular}{|c|c|c|c|c|c|c|c|c|c|}
\hline \multirow[t]{2}{*}{ ID } & \multirow{2}{*}{$\begin{array}{l}\text { CLC/GLC2000 } \\
\text { Vegetation class }\end{array}$} & \multirow{2}{*}{$\begin{array}{l}d \\
\left(\mathrm{~g} \mathrm{~m}^{-2}\right)\end{array}$} & \multicolumn{5}{|c|}{ Stand. emission potential $\left(\mu \mathrm{g} \mathrm{g}_{\mathrm{DW}}^{-1} \mathrm{hr}^{-1}\right)$} & \multirow{2}{*}{$\begin{array}{l}\text { Seas. } \\
\text { type }\end{array}$} & \multirow[t]{2}{*}{ Plant Composition ${ }^{\mathrm{c}}$} \\
\hline & & & ISOP & $\mathrm{MT}_{\text {synt }}$ & $\mathrm{MT}_{\text {pool }}$ & OVOC & SQT & & \\
\hline $117 \mathrm{a}$ & Cropland/Forest/Shrubland/Grass Mos. & 550 & 1.0 & 1.0 & 1.0 & 2.0 & 0.1 & eve & $\mathrm{o}$ \\
\hline $117 \mathrm{~b}$ & Cropland/Forest/Shrubland/Grass Mos. & 600 & 5.0 & 0.5 & 0.5 & 2.0 & 0.1 & eve & $\mathrm{p}$ \\
\hline $117 \mathrm{c}$ & Cropland/Forest/Shrubland/Grass Mos. & 370 & 5.0 & 0.5 & 0.5 & 2.0 & 0.1 & eve & $20 \%$ LC $12,80 \%$ LC 108 \\
\hline 125 & Cropland unspecified & 600 & 0.5 & 0.5 & 0.5 & 2.0 & 0.1 & dec & $30 \%$ LC $14,70 \%$ LC 18 \\
\hline 127 & Wetland - Bogs and Marshes & 330 & 10.0 & 1.0 & 1.0 & 2.0 & 0.1 & eve & LC 36 \\
\hline 128 & Wetland - Palsa Bogs & 170 & 10.0 & 1.0 & 1.0 & 2.0 & 0.1 & eve & LC $36,50 \%$ cover \\
\hline 129 & Wetland - Riparian Vegetation & 300 & 10.0 & 1.0 & 1.0 & 2.0 & 0.1 & eve & q \\
\hline 131 & Tree Cover, regularly flooded & 250 & 5.0 & 0.1 & 0.1 & 2.0 & 0.1 & eve & African Swamp \\
\hline
\end{tabular}

a Vegetation class ID of CLC/GLC2000 landcover mosaic as indicated in Köble (2007). b Seasonality types: eve=broadleaf/coniferous evergreen, dec=broadleaf/coniferous deciduous, mix $=50 \%$ evergreen, $50 \%$ deciduous, barl=spring and winter barley, maiz=fodder and grain maize. ${ }^{\mathrm{c}}$ LC is an abbreviation for CLC/GLC2000 land use class. ${ }^{\mathrm{d}} 15 \%$ Fagus sylvatica, 10\% Q. ilex, 10\% Betula pubescens, $10 \%$ Q. robur, $10 \%$ Q. pubescens, $10 \%$ Q. petraea, 20\% Eucalyptus, 5\% Juglans regia. ${ }^{\mathrm{e}} 40 \%$ Pinus sylvestris, $40 \%$ Picea abies, $20 \%$ Pinus pinea. f 25\% Q.ilex, 25\% Q. coccifera, 25\% Arbutus unedo, 25\% Myrtus communis. $\mathrm{g} 30 \%$ Q.ilex, $30 \%$ Q. coccifera, 30\% Arbutus unedo, $10 \%$ Myrtus communis. ${ }^{\mathrm{h}} 10 \%$ Arunda donax, $10 \%$ Vaccinum sp., 10\% Salix sp., $40 \%$ Betula pendula, $30 \%$ Fraxinus angustifolia. ${ }^{\mathrm{i}} 50 \%$ Betula pubescens, 30\% Populus tremula, 10\% Alnus glutinosa, 5\% Q. petrea, 5\% Tilia cordata. ${ }^{\mathrm{j}} 30 \%$ Fagus sylvatica, 20\% Betula pendula, 10\% Q. robur, $10 \%$ Q. pubescens, $10 \%$ Q. petraea, $10 \%$ Q. suber, $10 \%$ Q. ilex. ${ }^{\mathrm{k}} 30 \%$ Picea abies, $30 \%$ Pinus sylvestris, $10 \%$ Abies alba, $30 \%$ Betula pubescens. ${ }^{1}$ 50\% Pinus pinea, 50\% Pinus pinaster. ${ }^{\mathrm{m}} 15 \%$ Erica sp., $15 \%$ Vaccinum sp., $40 \% .{ }^{\mathrm{n}} 50 \%$ LC $101 \mathrm{c}, 10 \%$ Ulex parviflorus, $10 \%$ Myrtus communis, $10 \%$ Lavandula sp, 10\% Rosmarinus officinalis, $10 \%$ Nerium oleander. LC $101 \mathrm{~b}, 30 \%$ LC $36 .{ }^{\circ} 30 \%$ LC 12, 30\% LC 18, 20\% Pinus sylvestris, 20\% Betula pubescens. p 30\% LC 12, 30\% LC 18, 20\% Q. ilex, 20\% Pinus pinea. ${ }^{\mathrm{q}} 50 \%$ LC 36, 20\% Salix spp., 10\% Alnus cordata, 10\% Betula pendula, 10\% Populus alba.

(Fagus sylvatica L.) (Dindorf et al., 2006). Isoprene emissions depending on temperature and light were calculated based on the recent concept described in Guenther et al. (2006), from now on referred to as MEGAN (Model of Emissions of Gases and Aerosols from Nature). Additional factors controlling isoprene emissions as density of green leaves, soil moisture, and phenology of leaves can be taken into account in the MEGAN version 2 model by Guenther et al. (2006), but were not considered in this work. The main reason for choosing a simplified setup of the MEGAN model is to be comparable with the previous algorithm by Guenther (1997) that describes only temperature and light-dependence of emissions. The use of temperature and light dependent isoprene emission algorithms is supported by a recent study of Boissard et al. (2008) who demonstrated that, based on 25 emitter species and using varying environmental conditions $\left(10^{\circ} \mathrm{S}\right.$ to $\left.60^{\circ} \mathrm{N}\right)$, isoprene emissions are mainly sensitive ( $76 \%$ of the overall variability) to instantaneous temperature and photosynthetic photon flux density and to the cumulated 3 weeks air temperature.

The MEGAN canopy environment emission activity factor is:

$\gamma_{\mathrm{CE}, i}($ synt $)=\mathrm{C}_{\mathrm{CE}} \cdot \gamma_{\mathrm{T}} \cdot \gamma_{\mathrm{P}} \cdot \mathrm{LAI}$

For the canopy radiation model applied in this work, $C_{\mathrm{CE}}$ is set to 1.13 in order to obtain $\gamma_{\mathrm{CE}, i}($ synt$)=1$ at standard conditions. The value for $C_{\mathrm{CE}}$ is about 2 times larger than given in Guenther et al. (2006), where $C_{\mathrm{CE}}=0.57$ is used. This is due to the simplified canopy environment model applied in our work (see Sect. 2.4.4).
The temperature dependence of MEGAN is expressed as:

$\gamma_{T}=\frac{E_{\mathrm{opt}} \cdot C_{\mathrm{T} 2} \cdot \exp \left(C_{\mathrm{T} 1} \cdot x\right)}{C_{\mathrm{T} 2}-\left(C_{\mathrm{T} 1} \cdot\left[1-\exp \left(C_{\mathrm{T} 2} \cdot x\right)\right]\right)}$

with $x=\left(\frac{1}{T_{\mathrm{opt}}}-\frac{1}{T}\right) / R . \quad R$ is the universal gas constant $\left(R=0.00831 \mathrm{~kJ} \mathrm{~K}^{-1} \mathrm{~mol}^{-1}\right), C_{\mathrm{T} 1}, C_{\mathrm{T} 2}$ are empirical constants given by Guenther et al. (2006). $T$ is the instantaneous temperature (in $\mathrm{K}$ ). $E_{\text {opt }}$ is the maximum normalized emission capacity, and $T_{\mathrm{opt}}$ is the temperature at which Eopt occurs. These coefficients are estimated as a function of the average air temperature over the past $24 \mathrm{~h}$ (T24) and $240 \mathrm{~h}$ (T240):

$$
\begin{aligned}
E_{\mathrm{opt}} & =2.034 \cdot \exp (0.05 \cdot[\mathrm{T} 24-297]) \\
& \cdot \exp (0.05 \cdot[\mathrm{T} 240-297]) \\
& T_{\mathrm{opt}}=313+(0.6 \cdot[\mathrm{T} 240-297])
\end{aligned}
$$

In the work of Guenther et al. (2006), all temperatures are considered to be leaf temperatures calculated by a canopy environment model. Since our intention is to provide a BVOC emission model that can be readily implemented in 3-dimensional atmospheric transport models we reduced the complexity of the emission calculation by using air temperature instead of leaf temperature. The light dependence of MEGAN is given by:

$\gamma_{P}=\frac{\alpha_{m} C_{p} L}{\sqrt{1+\alpha_{m}^{2} L^{2}}}$ 
The empirical coefficients in Eq. (13), $\alpha_{m}$ and $C_{p}$, depend on the past history of light levels. These parameters are calculated as:

$$
\begin{aligned}
& \quad \alpha_{m}=0.004-0.0005 \cdot \ln (\mathrm{P} 240) \\
& \quad C_{p}=0.0468 \cdot \exp \left(0.0005 \cdot\left[\mathrm{P} 24-\mathrm{P}_{0}\right]\right)(\mathrm{P} 240)^{0.6}
\end{aligned}
$$

P24 and P240 are the photosynthetically active photon flux density (PPFD) averages over the past 24 and $240 \mathrm{~h}$, respectively. $\quad P_{0}$ equals $200 \mu \mathrm{mol} \mathrm{m}^{-2} \mathrm{~s}^{-1}$ for sunlit leaves and $50 \mu \mathrm{mol} \mathrm{m}^{-2} \mathrm{~s}^{-1}$ for shaded leaves.

Monoterpene emissions depending on temperature and light were calculated based on Guenther (1997). This approach was also applied to calculate an alternative isoprene emission estimate. The emission activity factor for the direct emissions of these newly synthesized compounds is according to Guenther (1997):

$\gamma_{\mathrm{CE}, i}($ synt $)=\gamma_{T} \cdot \gamma_{P} \cdot \gamma_{\text {seas }}$

with

$$
\begin{aligned}
\gamma_{T} & =\frac{\exp \left(\left[C_{\mathrm{T} 1}\left(T-T_{S}\right)\right] / R T_{S} T\right)}{C_{\mathrm{T} 3}+\exp \left(\left[C_{\mathrm{T} 2}\left(T-T_{M}\right)\right] / R T_{S} T\right)} \\
\gamma_{P} & =\frac{\alpha C_{\mathrm{L} 1} L}{\sqrt{1+\alpha^{2} L^{2}}}
\end{aligned}
$$

Here $L$ is PPFD (in $\mu$ mol photons $\mathrm{m}^{-2} \mathrm{~s}^{-1}$ ), $R$ is the universal gas constant, and $C_{\mathrm{L} 1}, C_{\mathrm{T} 1}, C_{\mathrm{T} 2}, C_{\mathrm{T} 3}, \alpha$ and $T_{M}$ are empirical constants given by Guenther (1997). The approach of calculating isoprene and monoterpene emissions presented with Eqs. (15-17) is termed G97 in the following.

\subsubsection{Seasonal variability of emissions}

Seasonality of emissions (non-temperature and non-light) accounts for several characteristic observations:

1. Development stage of leaves on deciduous trees has an impact on their emission capacity. Emissions from deciduous vegetation are reduced or become negligible during winter dormancy and due to loss of leaves.

2. Emissions from agriculture are limited to the growing season. Exceptions are tomatoes and other fruits and vegetables that are cultivated in greenhouses.

3. Emissions from evergreen vegetation occur during the whole year and have a seasonal variation.

4. Snow coverage prevents emissions from all vegetation types. Though there might be emission from noncovered parts of a coniferous tree, it is assumed that these are close to zero and negligible.

Several vegetation types with different seasonality of emissions due to leaf phenology and growing season were defined: seven agriculture crop types with different growing seasons, deciduous vegetation type (broadleaf and coniferous trees, wetlands and other deciduous vegetation) and evergreen vegetation type (broadleaf and coniferous trees, grassland, pastures and other evergreen vegetation).

Seasonality of emissions is derived from the leaf coverage per unit ground area, expressed by the leaf area index (LAI), in combination with data on growing season for crops and leaf-on season for deciduous trees. MODIS-based LAI data provides information regarding the evolution and structure of seasonal vegetation characteristics (Zhang et al., 2004). MODIS LAI data with $1 \mathrm{~km}$ horizontal resolution is retrieved for the years 2004/2005 from Boston University (ftp: //primavera.bu.edu/pub/datasets/MODIS/MOD15_BU/) and averaged over the model grid, i.e. the total LAI of a model grid cell corresponds to one MODIS derived LAI value. This LAI value is used to calculate the actual LAI of each vegetation type $v$ (see below) and by scaling emissions with the vegetation type LAI the seasonal variability of emissions with respect to growing season length and development stages of leaves is simulated. A phenology correction factor $p$ is introduced into the model with values between 0 and 1 , with $p=0$ during no emission months and $p=1$ during full emission months.

Growing season dates (emergence, subsequent growth stages, harvest) for seven different crops, i.e. maize (fodder and grain maize), potato, rape seed (spring and winter), wheat, (spring and winter), sugar beet, sunflower and barley (spring and winter), have been obatined from Bouraoui and Aloe (2007). All other agriculture crops have been attributed to one of these seven crop phenology types (see Table 4). The crop growing season is defined by the time period between emergence and harvest. The harvest month was chosen as end date for emissions. Starting from the date of emergence, $p$ is linearly increasing from 0 to 1 , i.e. from growing seedlings to fully developed plants. The $p$ of fully developed plants is set to 1 , while after harvest $p$ is 0 . Monthly crop phenology data with a horizontal resolution of $50 \mathrm{~km}$ was used to derive an average $p$ value for the seven crop types in each bioclimatic zone. Greenhouse tomatoes and plantation crops are treated as non-seasonal crops ( $p=1$ all year).

Deciduous trees lose their leaves in winter. With the development of new leaves in spring, emissions are retarded. For deciduous and evergreen vegetation types, the seasonal variation of the phenology correction factor has the form of a bell shape with maximum in July, and can be calculated following Staudt et al. (2000):

$p=1-\rho\left(1-\exp \left[\left(M-M_{0}\right) / \tau\right]\right)$

With $M$ being the month of the calendar year, $M_{0}=7$ (July), $\rho$ determines the relative amplitude, and $\tau$ is the length of the active emission period in months. For deciduous vegetation, $\rho$ is set to 1 , considering that emissions are zero during a dormancy period. The latitudinal separation of the growing season length is the same as in the NatAir inventory (Steinbrecher et al., 2009): 
$>65^{\circ} \mathrm{N}$ and in the arctic zone: $\tau=3$; from $50-65^{\circ} \mathrm{N}: \tau=5$; between $40-50^{\circ} \mathrm{N}: \tau=6$; and in Southern Europe at latitude $<40^{\circ} \mathrm{N}: \tau=8$.

For evergreen coniferous trees, evergreen broadleaf trees, other evergreen vegetation, and grassland (see Table 5), Eq. (18) is used with the parameters $\rho=0.8$ and $\tau=12$ in all geographic zones.

The total LAI in a grid cell $\left(\mathrm{LAI}_{\mathrm{tot}, i}\right)$ is the sum of the LAI contributions of each vegetation type $v$ (seven agriculture types, deciduous and evergreen), $\mathrm{LAI}_{v, i}$. Total LAI by definition equals the MODIS LAI (LAI $\mathrm{Lodis}, i_{i}$ ) in that cell:

$\mathrm{LAI}_{\text {tot }, i}=\mathrm{LAI}_{\text {modis }, i}=\sum_{v} \mathrm{LAI}_{v, i}$

LAI values for each vegetation phenology type $\mathrm{LAI}_{v}$ in a grid cell are calculated from the MODIS LAI of the cell considering the respective phenology correction $p_{v}$ and area fraction $a_{v}$. For a grid cell that contains wheat (index crop), deciduous (index dec) and evergreen coniferous (index eve) trees, the respective $\mathrm{LAI}_{\text {crop }}$ of wheat would be:

$\mathrm{LAI}_{\text {crop }}=\frac{a_{\text {crop }} \cdot p_{\text {crop }} \cdot \mathrm{LAI}_{\text {modis }}}{a_{\text {crop }} \cdot p_{\text {crop }}+a_{\text {dec }} \cdot p_{\text {dec }}+a_{\text {eve }} \cdot p_{\text {eve }}}$

LAI for all other vegetation types are calculated in analogy to Eq. (20). To illustrate the effectiveness of this approach, we consider a grid cell containing area fractions of $40 \%$ wheat, $40 \%$ deciduous trees, and $20 \%$ evergreen coniferous trees at $45^{\circ} \mathrm{N}$. January: MODIS LAI is 0 indicating snow coverage; phenology correction is $p_{\text {crop }}=0$ and $p_{\text {dec }}=0.37$, and $p_{\text {eve }}=0.69$; then all vegetation type LAI are 0 . March: MODIS LAI is 2; phenology correction is $p_{\text {crop }}$ is 0.25 (emerging seedlings) and $p_{\text {dec }}$ is 0.51 , and $p_{\text {eve }}$ is 0.77 . Thus emissions from crop and deciduous trees are about to evolve while evergreen trees almost emit fully; then the calculated respective $\mathrm{LAI}$ are: $\mathrm{LAI}_{\text {crop }}=0.43, \mathrm{LAI}_{\mathrm{dec}}=0.89$, and $\mathrm{LAI}_{\text {eve }}=0.67$. July: MODIS LAI is 6 , all vegetation types are emitting; all $p$ values are 1 and the respective vegetation type $\mathrm{LAI}$ are $\mathrm{LAI}_{\text {crop }}=2.4, \mathrm{LAI}_{\mathrm{dec}}=2.4$, and $\mathrm{LAI}_{\text {eve }}=1.2$.

To calculate seasonal canopy emission activities $\gamma_{\mathrm{CE}, i}$ (synt) for different vegetation types in the MEGAN model, the specific vegetation type LAI of the grid cell obtained from Eq. (20) are inserted into Eq. (10). In the G97 model the seasonality of emissions from each vegetation type is taken into account by using the seasonality factor $\gamma_{\text {seas }}$ (see Eqs. 8 and 15) together with the specific vegetation type LAI:

$\gamma_{\text {seas }}=\frac{0.49 \mathrm{LAI}_{v}}{\sqrt{1+0.2 \mathrm{LAI}_{v}^{2}}}$

This approach consequently leads to a scaling of the emission from a certain vegetation type during the months when the emissions are not at the full extent due to phenology of leaves. The seasonality factor ensures that there are zero emissions from deciduous trees in wintertime when leaves are not present. Emissions from agriculture occur only during the growing season. This approach also ensures that in case of a completely snow covered surface in a grid cell (i.e. monthly average $\mathrm{LAI}_{\mathrm{tot}, i}=0$ ), emissions from all vegetation types are set to zero.

\subsubsection{Setup of the canopy environment model}

The BVOC emission model applies the radiation code of Weiss and Norman (1985) to calculate the extinction of PPFD as a function of the leaf area index, the distribution of the LAI (leaf area density) inside the canopy, the fraction of leaves and the orientation of these leaves. The canopy is divided into several vertical layers and each layer distinguishes between shaded and sunlit parts of the canopy. The division into shaded and sunlit leaves takes into account the actual solar angle and the foliage density described by the LAI. While PPFD is calculated for each vertical canopy layer, the leaf temperature is simply replaced with air temperature and is uniform for the whole canopy in order to reduce the computational demand for calculation of European BVOC emissions.

The canopy environment model was carefully checked to obtain canopy environment emission activity factors $\left(\gamma_{\mathrm{CE}, i}(\mathrm{synt})\right)$ with values close to unity at standard conditions for both the G97 and the MEGAN version. For G97, $\gamma_{\mathrm{CE}, i}$ (synt) is 0.991 at standard conditions given in Guenther (1997) and for MEGAN $\gamma_{\mathrm{CE}, i}$ (synt) is 0.997 at the standard conditions mentioned in Guenther et al. (2006).

For each grid cell $i$ of the domain in addition to the emitting area $A_{i}$, foliar biomass density $D_{i}$, BVOC emission factors $\epsilon_{i, b}$, meteorological maps of temperature and light intensity are required (see Fig. 1). The meteorological data of $2 \mathrm{~m}$ air temperature (in $\mathrm{K}$ ) and solar surface radiation (in $\mathrm{W} \mathrm{m}^{-2}$ ) from ECMWF are the drivers used as input for the BVOC emission model. The meteorological data is used with a horizontal resolution of $1 \times 1$ degrees.

\section{Results}

Monthly averaged BVOC emission rates for Europe were computed for the two years 2004 and 2005 according to Eq. (1). All calculations were performed with a temporal resolution of $3 \mathrm{~h}$ and a spatial resolution of $0.089 \times 0.089 \mathrm{de}-$ grees. Two years were selected in order to obtain a more reliable basis for studying seasonal changes, rather than to investigate inter-annual variability of emissions. The years 2004 and 2005 were both relatively warm years in Europe with mean annual temperatures $0.5-0.6 \mathrm{~K}$ higher than in the reference period 1961-1990. The canopy environment model described in Sect. 2.4 was applied to calculate emissions of monoterpenes, sesquiterpenes and OVOC according to the emission algorithms of Guenther (1997). Isoprene emissions 

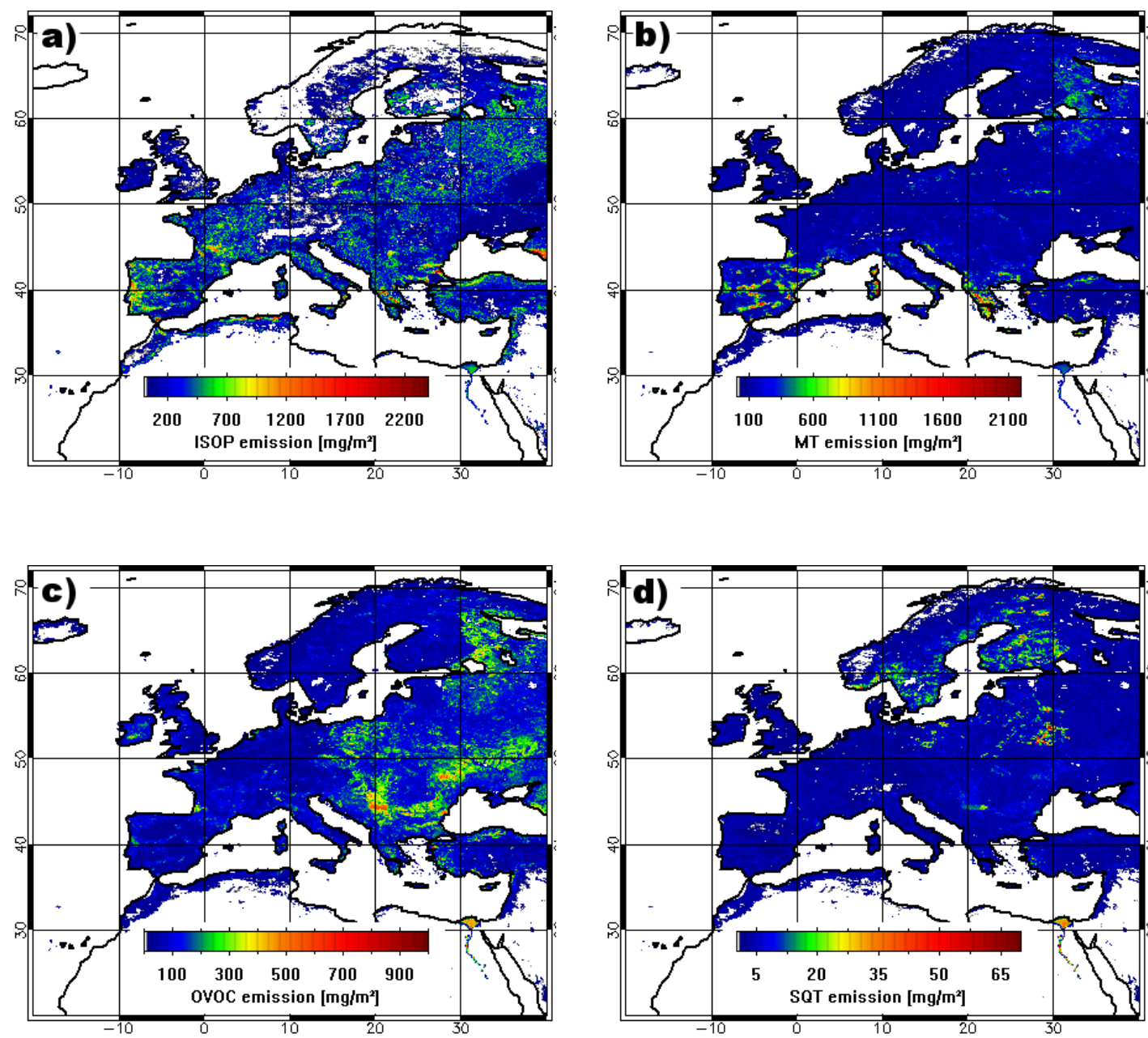

Fig. 2. European emission (in $\mathrm{mg} \mathrm{m}^{-2}$ ) distribution for July 2005 of: (a) isoprene, (b) monoterpenes, (c) OVOC, (d) sesquiterpenes. Isoprene emissions are calculated with MEGAN. The maximum differences for July 2005 between the isoprene emission distribution calculated with MEGAN and G97 is only 6\%. Emissions shown here are assessed with the assumption that terpenes and other VOC emitted by plants are directly emitted into the atmosphere, ignoring eventual oxidation and losses within the canopy.

were obtained using the temperature and light dependence of the new MEGAN algorithm. In addition, isoprene emissions were computed with G97.

Section 3.1 presents results on BVOC emissions for $\mathrm{Eu}-$ rope and the major sources of each compound class. The composition and amount of BVOC emissions from different land use types in the four main vegetation zones of Europe is analysed in Sect. 3.2. The seasonality of emissions is described in Sect. 3.3. In Sect. 3.4 the seasonal behaviour and the major contributions to the emissions are investigated for four European countries. Results for these countries are compared with previous national inventories. Finally, we compare the total BVOC emission amounts obtained in our emission inventory to previous European emission estimates in Sect. 3.5.

\subsection{European BVOC emissions}

The average annual total BVOC emission obtained from the two years 2004 and 2005 for the Pan-European domain is $12 \mathrm{Tg}$. Calculated annual emissions from both years are very similar: $11.6 \mathrm{Tg}$ in 2004 and $12.3 \mathrm{Tg}$ in 2005 . The OVOC class which represents mainly oxygenated VOC contributes about $4.5 \mathrm{Tg}$ and monoterpenes about $4 \mathrm{Tg}$ to the total BVOC emissions. Annual isoprene emissions are found to be about 3.5 Tg, independent of the chosen emission algorithm.

Agriculture, forests and other land use areas cover $40.4 \%$, $34.5 \%$ and $25.1 \%$ of the vegetated surface of Pan-Europe, respectively. Forests contribute with $55 \%$ and agriculture contributes with $27 \%$ to the total BVOC emissions, the remaining emissions come from other land use types like shrubs, grassland and wetlands. The contribution of agriculture 

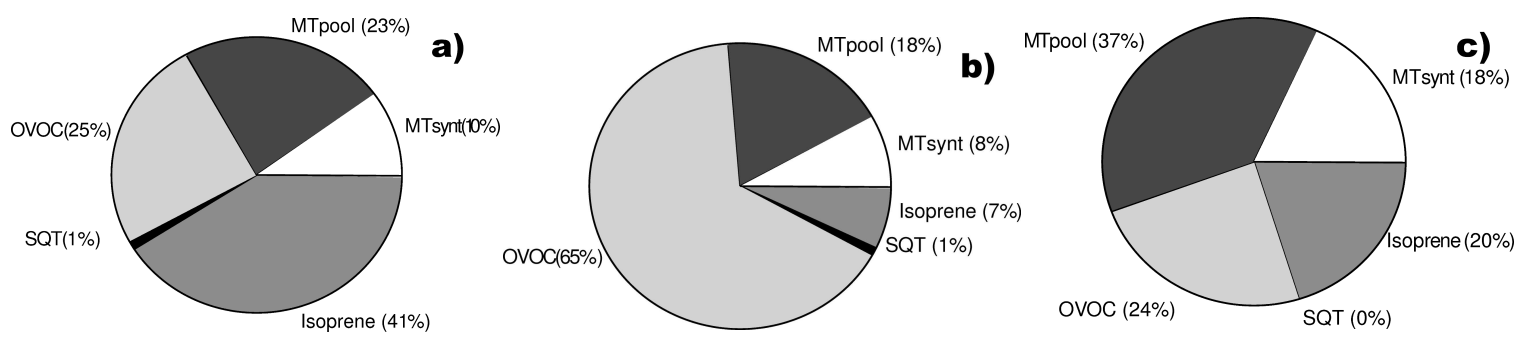

Fig. 3. European BVOC class emission contributions to each land use type: (a) forest (b) agriculture (c) other land use.

in the presented European emission inventory is somewhat higher than in the European inventory of Simpson et al. (1999) who have attributed $20 \%$ of the total European BVOC emissions to agriculture.

The European distribution of isoprene emissions for July 2005 calculated with the MEGAN emission algorithm is shown in Fig. 2a. Isoprene emissions calculated with the G97 algorithm for the same summer month were found to be up to $10 \%$ lower than the MEGAN isoprene estimate in Pan-Europe. The largest differences are located in Northern Africa, where the monthly temperature average was above $30^{\circ} \mathrm{C}$ and mean solar surface radiation was above $240 \mathrm{~W} \mathrm{~m}^{-2}$. We thus assume that the differences are due to the different parameterization of the temperature and light dependence. Highest isoprene emission rates are found in the Mediterranean region both in summer and winter. In July 2005 up to $2000 \mathrm{mg} \mathrm{m}^{-2}$ of isoprene is emitted from the Mediterranean vegetation in certain locations. Forests are the major source of isoprene and contribute $80 \%$ to the emitted amount of isoprene. Isoprene emissions constitute $28 \%$ of the total emitted BVOC amount in Europe.

Newly synthesized monoterpene ( $\left.\mathrm{MT}_{\text {synt }}\right)$ emissions contribute $31 \%$ to the annual emitted amount of monoterpenes in Europe. Though the emission rate of $\mathrm{MT}_{\text {synt }}$ can regionally be higher than that of $\mathrm{MT}_{\text {pool }}$ (see Fig. A3, Electronic Supplement: http://www.biogeosciences.net/6/1059/2009/ bg-6-1059-2009-supplement.pdf), prevalent solar radiation levels in Europe inhibit a more intense release of $\mathrm{MT}_{\text {synt }}$. The monthly averaged $\mathrm{MT}_{\text {synt }}$ emission rate in July 2005 is below $100 \mathrm{mg} \mathrm{m}^{-2}$ in Sweden and Finland, while it is much higher, $800-1200 \mathrm{mg} \mathrm{m}^{-2}$, in Spain, Italy and Greece (Fig. A3b, Electronic Supplement: http://www.biogeosciences.net/6/ 1059/2009/bg-6-1059-2009-supplement.pdf). Figure 2b shows the distribution of monoterpene emissions for July 2005. Apart from some regions with elevated monoterpene emissions, like Spain, Corsica and Greece, the emission rates are below $1000 \mathrm{mg} \mathrm{m}^{-2}$. Relatively high monoterpene emission rates can be found over Scandinavia and the Baltic countries which are covered with large boreal coniferous forests. In the boreal region, monoterpene emissions constitute about $50 \%$ of the forest emissions. The major fraction of monoterpene emissions in Europe stem from forests.
European Maps of BVOC emission rates for July 2005 of OVOC and sesquiterpenes are presented in Fig. $2 \mathrm{c}$ and $2 \mathrm{~d}$. Half of the emitted amount of the OVOC class originates from agriculture and OVOC emission rates are highest over regions with intensive agriculture in Eastern Europe (Fig. 2c) with up to $800 \mathrm{mg} \mathrm{m}^{-2}$ in July 2005. Crops with highest foliar biomass density and land coverage will exhibit highest OVOC emissions. It can be deduced that elevated OVOC emissions in Western Europe are from maize, common wheat and other cereals, each covering $10-50 \%$ of the area in Western France and the Netherlands. Maize is the crop species with the highest foliar biomass density (see Table 4).

Sesquiterpenes contribute only $1 \%$ to the total emitted amount. However this class of compounds is of great interest for estimating the formation of secondary organic aerosol. Forests are the largest source of sesquiterpenes and the centre of summertime SQT emissions is in Northeastern Europe, mainly Sweden, Finland and the European part of Russia (Fig. 2d). The Nile Delta is a region with relatively high sesquiterpene ( $35 \mathrm{mg} \mathrm{m}^{-2}$ ), OVOC $\left(500 \mathrm{mg} \mathrm{m}^{-2}\right.$ ) and newly synthesized monoterpene $\left(900 \mathrm{mg} \mathrm{m}^{-2}\right)$ emissions in July 2005. This region is covered by $100 \%$ with the GLC vegetation class of irrigated cropland. The Nile Delta is thus one example how inaccurate, not crop-specific, land use information can affect emission rates calculated in this inventory.

The contribution of different BVOC classes to the European emissions from forests, agriculture and other land use are presented in Fig. 3a-c. Isoprene dominates European forest emissions, while the OVOC class contributes a fourth and the monoterpenes about a third to the forest emissions. European other land use vegetation predominantly emits monoterpenes. BVOC emissions from agriculture in Europe mainly consist of OVOCs. For the major vegetation zones of Europe annual amount and composition of BVOC emissions from different land use types are analysed in the next section.

\subsection{Emissions from major vegetation zones}

The European Union is covered with four major vegetation zones: Atlantic (Western Europe), Continental (Central and Eastern Europe), Boreal (Scandinavia and Northeastern Europe) and Mediterranean. The annual average amounts obtained from the 2004 and 2005 simulations of each BVOC 
Table 6. 2004-2005 annual averaged BVOC emissions for the four major vegetation zones in Europe from the presented inventory. For the main land use types agriculture, forests and other land use and for the total land use, annual emissions (in Gg) are shown for each BVOC class in the four vegetation zones Atlantic, Boreal, Continental, and Mediterranean and for the total of these four zones.

\begin{tabular}{llrrrrr}
\hline Land Use type & BVOC class & Atlantic & Boreal & Continental & Mediterranean & Total \\
\hline Agriculture & ISOP & 15 & 8 & 51 & 12 & 86 \\
& MT $_{\text {synt }}$ & 28 & 14 & 128 & 19 & 189 \\
& MT pool & 35 & 29 & 151 & 18 & 233 \\
& OVOC & 141 & 76 & 538 & 69 & 824 \\
& SQT & 2 & 1 & 9 & 2 & 14 \\
Forest & Total & 221 & 128 & 877 & 119 & 1346 \\
& ISOP & 186 & 350 & 382 & 235 & 1154 \\
& MT & 41 & 354 & 141 & 88 & 625 \\
& MT & 23 & 494 & 89 & 63 & 668 \\
& OVOC & 31 & 461 & 140 & 64 & 695 \\
& SQT & 1 & 21 & 6 & 1 & 29 \\
Other Land Use & Total & 282 & 1680 & 759 & 451 & 3172 \\
& ISOP & 27 & 20 & 14 & 95 & 155 \\
& MT & 10 & 12 & 11 & 130 & 163 \\
& MT & 23 & 69 & 10 & 210 & 311 \\
& OVOC & 32 & 44 & 11 & 70 & 157 \\
& SQT & 0.4 & 1 & 0.2 & 2 & 3 \\
& Total & 92 & 145 & 46 & 507 & 789 \\
\hline Total Land Use & Total & $\mathbf{5 9 5}$ & $\mathbf{1 9 5 3}$ & $\mathbf{1 6 8 2}$ & $\mathbf{1 0 7 7}$ & $\mathbf{5 3 0 7}$ \\
\hline
\end{tabular}

class emitted from agriculture, forest and other land use for these vegetation zones are given in Table 6. BVOC emissions sum up to $1.3 \mathrm{Tg}$ from agriculture, $3.2 \mathrm{Tg}$ from forests and $0.8 \mathrm{Tg}$ from other land use.

Emissions in the Atlantic zone are mainly from forests and agriculture (Table 6). Agriculture emissions mainly consist of OVOCs, while the forest emissions mainly consist of isoprene. Fruits cover $10-20 \%$ of the agriculture area in the Atlantic zone. There are some hot spot regions for the cultivation of tomatoes and other vegetables in Western France and Netherlands with area fractions of up to $40 \%$. Widespread crops like wheat, maize, rape, and cereals have been assigned MT standard emission factors of $0.5 \mu \mathrm{gg}_{\text {DW }}^{-1} \mathrm{~h}^{-1}$ each for pool and newly synthesized MT emissions. The uncertainties associated with agriculture emissions are further discussed in Sect. 4.3.

About $90 \%$ of the emissions of the Boreal zone are from forests. Monoterpene emissions contribute $50 \%$ and isoprene emissions contribute $20 \%$ to the BVOC emissions in forests of the Boreal zone. It should be noted that emissions from Picea abies in the Boreal zone are reduced by $40 \%$ compared to the Contintental zone due to the bioclimatic correction of the foliar biomass density. Despite this reduction total BVOC emissions from boreal forest are about two times the continental forest emissions.

Forest and agriculture contribute almost equally to BVOC emissions from the Continental zone. For continental forests it is interesting to note that a higher fraction of the monoterpene emissions originate from the newly synthesized emis- sion route. This is certainly due to the dominance of European beech (Fagus sylvatica). Forest BVOC emissions in the Continental zone consist of $18 \%$ OVOC, $50 \%$ isoprene, $30 \%$ monoterpenes and $1 \%$ sesquiterpenes. Isoprene emissions from continental forests amount to $0.4 \mathrm{Tg}$.

Monoterpene emissions contribute $33 \%$ to the BVOC emissions in forests of the Mediterranean region. These emissions can largely be attributed to the pool MT emissions from Pinus pinaster and to newly synthesized MT emissions from Quercus ilex (standard emission factor of $43 \mu \mathrm{gg}_{\mathrm{DW}}^{-1} \mathrm{~h}^{-1}$ ). Isoprene emissions constitute $52 \%$ of the Mediterranean forest emissions and mainly originate from the Mediterranean oaks. Emissions from other land use vegetation contribute substantially (with 47\%) to the BVOC amount emitted in the Mediterranean region. BVOC emissions from other land use and from forests in the Mediterranean each amount to about $0.5 \mathrm{Tg}$.

\subsection{Seasonality}

The period from May to September is the time with enhanced BVOC emissions (Fig. 4). During this period ca. $80 \%$ of the total annual BVOC amount is released from vegetation. In April deciduous trees start to develop leaves and the increasing density of foliage of deciduous trees leads to a strong increase of BVOC emissions from March to April and from April to May. Between April and May emissions of isoprene, OVOC and monoterpenes more than double and SQT emissions increase by a factor of 3 . From June to August 


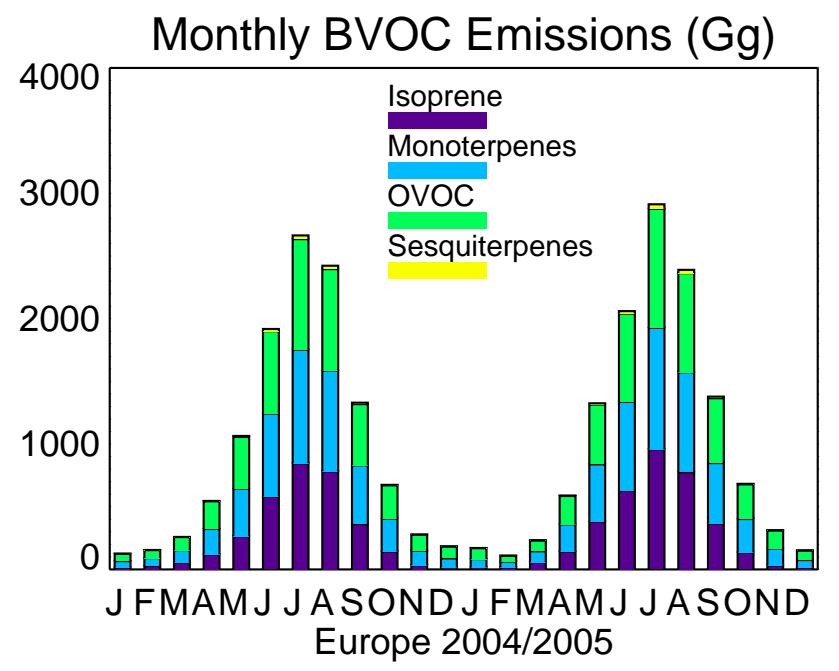

Fig. 4. Seasonal variation of European BVOC emissions.

emissions from vegetation are highest since light intensities, temperatures and emitting plant biomass are highest during these months. After September emissions decline rapidly due to the loss of leaves from deciduous trees and due to the end of the growing season for most agriculture crops. From November to March the monthly emitted amount of OVOC and MT compounds remains essentially constant while isoprene emission amounts show a strong dependence on the available light intensity.

The seasonal variation of contributions from the main land use types (agriculture, forests, other land use) to the emission of BVOC is shown in Fig. 5. In summer, forests are the main source of biogenic VOC, due to their high density of available foliar biomass for emitting these compounds. As shown in Fig. 3a, isoprene is the most abundant compound in European forest emissions. The contribution from the three main land use types becomes almost equal during the winter months (November to March).

In Fig. 6 the different seasonality of isoprene emissions calculated either using the MEGAN or the G97 algorithm is shown. The seasonal cycle of isoprene emissions calculated with MEGAN and G97 both resemble the shape of a Gaussian distribution with a maximum in July. The seasonal isoprene emission cycle calculated with the G97 emission algorithms is very similar to the MEGAN result.

During the summer months, June-August, MEGAN tends to give higher isoprene amounts, while during winter months isoprene emissions from MEGAN are in general lower than those from G97. The relative difference between the two algorithms is not larger than $35 \%$ for the studied months, and most likely caused by the different parameterisation of the light dependence and the different weighting of the foliar biomass influence. MEGAN takes into account the influence of light and temperature conditions during the previous day(s) and when warm and sunny conditions have occured

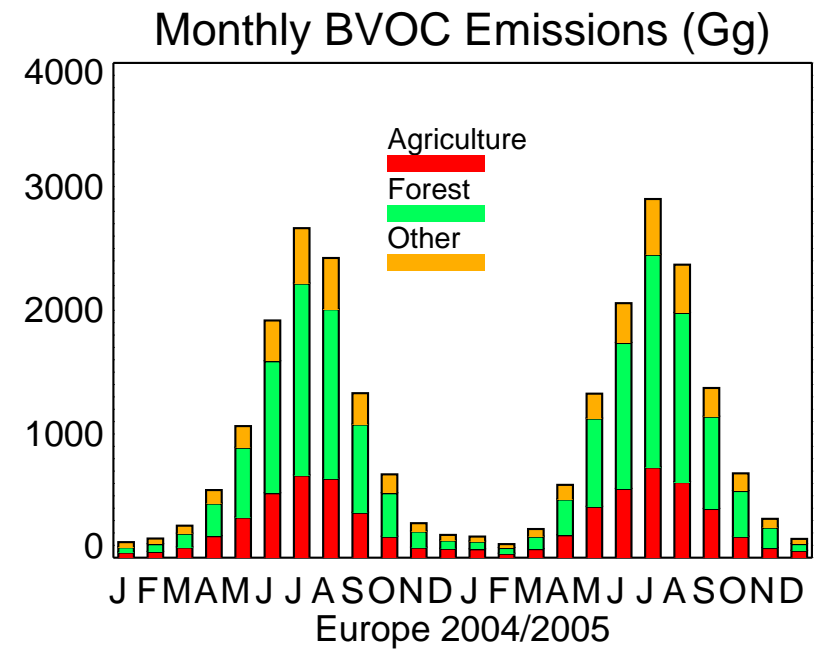

Fig. 5. Seasonal variation of the land use contributions to European total BVOC emissions.

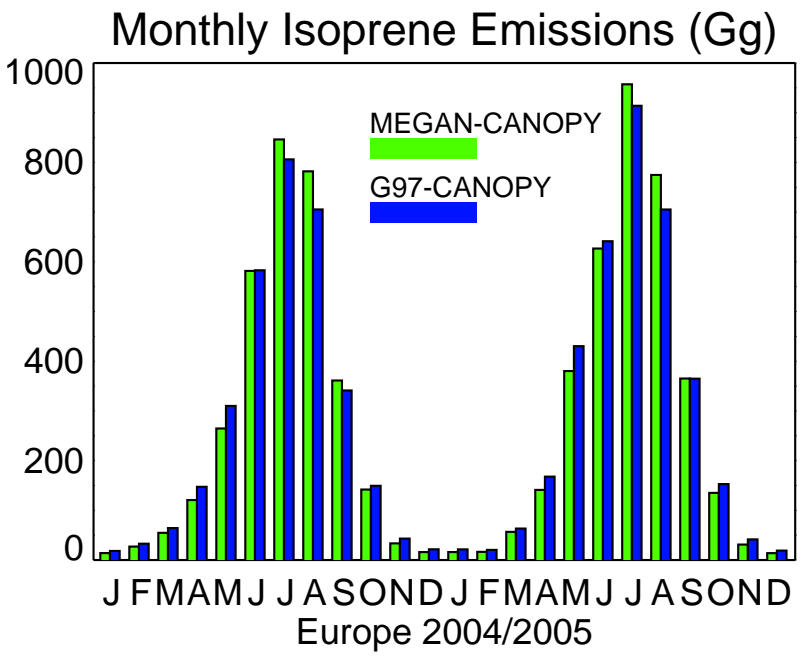

Fig. 6. Seasonal variation of European isoprene emissions.

prior to the time of observation, emission rates of isoprene are predicted to be enhanced. A further important difference between the two isoprene emission algorithms is the treatment of foliar biomass seasonality. The canopy emission acitivity is considered to be a linear function of the LAI in the MEGAN parameterisation (Eq. 10), while the seasonality factor used in G97, $\gamma_{\text {seas }}$, has a weaker dependence on LAI (Eq. 21). It is noted that the canopy average $\gamma_{P}$ in Eq. (10) (for MEGAN) and in Eq. (15) (for G97) also varies with LAI and thus the overall response to LAI in both models is less than linear. 
Table 7. Country-specific BVOC emissions from the presented inventory for Spain, Great Britain, Finland and Germany, calculated for the year 2005. Seasonal emissions are given as 3-monthly emission sum (in Gg) for the main land use types agriculture, forests and other land uses and for the total land use. The annual emission amount (in $\mathrm{Gg}$ ) is compared to the country-specific emission estimate given by Simpson et al. (1999) for each land use. (DJF: December to February, MAM: March to May, JJA: June to August, SON: September to November).

\begin{tabular}{|c|c|c|c|c|c|c|c|}
\hline Region & Land Use & DJF & MAM & JJA & SON & Total & Simpson et al. (1999) \\
\hline \multicolumn{8}{|l|}{ Spain } \\
\hline & Agriculture & 5 & 19 & 45 & 17 & 87 & 87 \\
\hline & Forest & 19 & 77 & 265 & 82 & 444 & 511 \\
\hline & Other Land Use & 32 & 104 & 278 & 97 & 511 & 222 \\
\hline & Total & 57 & 200 & 588 & 196 & 1042 & 820 \\
\hline \multicolumn{8}{|c|}{ Great Britain } \\
\hline & Agriculture & 15 & 22 & 50 & 26 & 113 & 76 \\
\hline & Forest & 4 & 7 & 21 & 9 & 40 & 77 \\
\hline & Other Land Use & 8 & 11 & 31 & 16 & 65 & 25 \\
\hline & Total & 27 & 40 & 102 & 51 & 219 & 178 \\
\hline \multicolumn{8}{|l|}{ Finland } \\
\hline & Agriculture & 0 & 1 & 5 & 2 & 8 & 9 \\
\hline & Forest & 5 & 44 & 266 & 88 & 404 & 341 \\
\hline & Other Land Use & 1 & 18 & 112 & 35 & 166 & 5 \\
\hline & Total & 6 & 63 & 383 & 125 & 577 & 355 \\
\hline \multicolumn{8}{|c|}{ Germany } \\
\hline & Agriculture & 7 & 24 & 64 & 28 & 123 & 66 \\
\hline & Forest & 10 & 40 & 123 & 45 & 217 & 377 \\
\hline & Other Land Use & 0 & 2 & 5 & 2 & 9 & 31 \\
\hline & Total & 17 & 66 & 191 & 75 & 349 & 474 \\
\hline
\end{tabular}

\subsection{Country-specific emission patterns}

Four countries within Europe, each representing a different vegetation zone, were selected to investigate the seasonal variation of BVOC emissions and the contribution of different land use types on a regional scale. Emitted BVOC amounts from different land use types are compared to other country-specific estimates from earlier inventories.

Spain is intended to represent the Mediterranean zone with forests dominated by Pinus pinaster or Quercus ilex. Great Britain was chosen to represent the Atlantic vegetation zone and is covered by non-irrigated arable land, agricultural crops, deciduous and evergreen forests. In Scotland a high fraction of other land use, moors and heathland, can be found. Finland represents the Boreal zone and boreal coniferous forests cover about $60 \%$ of the land area and the dominant tree species are Scots pine (Pinus sylvestris) and Norway spruce (Picea abies). Germany is in the Continental zone, which is used in this work as the reference zone to define bioclimatic correction factors. Northern Germany is dominated by agriculture, while the landscape in Southern Germany is characterised by a mosaic of mixed forests with Fagus sylvatica and Picea abies as major tree species and agriculture.

Seasonal BVOC emissions for the year 2005 are displayed in Table 7 and country-specific BVOC emissions are given in $\mathrm{Gg}$ for each country. BVOC emissions for the year 2005 are highest in Spain and sum up to about $1 \mathrm{Tg}$. Emissions from the vegetation in Germany are a factor of 3 lower than in Spain. The emitted amounts for Spain, Germany and Great Britain are within $30 \%$ of the national estimates given in the European inventory by Simpson et al. (1999). Our emission amount for Finland is roughly $60 \%$ higher than the estimate of Simpson et al. (1999) due to higher emissions from other land use types in our inventory (see Table 7).

Half of the BVOC emissions in Spain originate from other land use types like Mediterranean shrubland. Forests are also a very important source, while emissions from agriculture play a minor role in Spain. From spring (March to May, MAM) to summer (June to August, JJA) BVOC emissions from forests and other land use types increase by a factor of 3 , while emissions from agriculture increase less. Isoprene contributes $65 \%$ to the summer emissions from forests. Isoprene emission from forests sum up to $267 \mathrm{Gg}$ per year, which is $50 \%$ higher than the previous estimate given by Simpson et al. (1999). The BVOC emission amount for forests and agriculture is in good agreement with Simpson et al. (1999) while the other land use emission amount is a factor of 2 higher than the estimate given by Simpson et al. (1999).

One half of the BVOC emissions in Great Britain are from agriculture, the other half is from forests and other land use. Emission from forests contribute only one fifth to the total BVOC emissions. Agriculture emissions in summer (JJA) are two times higher than in spring and in autumn (September 
Table 8. Comparison with other European BVOC emission estimates from bottom-up inventories. Estimates from global emission inventories are included for the sake of completeness.

\begin{tabular}{llllll}
\hline $\begin{array}{l}\text { Total BVOC } \\
\mathrm{Gg} \mathrm{yr}^{\mathrm{a}}\end{array}$ & \begin{tabular}{l} 
Forest BVOC $^{\mathrm{b}} \mathrm{Gg} \mathrm{yr}^{-1}$ \\
\hline 12
\end{tabular} & $\begin{array}{l}\text { ISOP } \\
\mathrm{Gg} \mathrm{yr}^{-1}\end{array}$ & Reference & Inventory & Extent to East \\
& 6.5 & 3.4 & This Work, G97 & This Work & This Work \\
& & 3.4 & This Work, MEGAN & GEIA (Guenther et al., 1995) & $40^{\circ} \mathrm{E}$ \\
& & 10.3 & This Work, G97 & $36^{\circ} \mathrm{E}$ \\
$15-25$ & $9-15$ & 4.5 & This Work, MEGAN & MEGAN (Guenther et al., 2006) & $36^{\circ} \mathrm{E}$ \\
34 & & $4-5$ & Steinbrecher et al. (2009) & NatAir & $40^{\circ} \mathrm{E}$ \\
13.5 & 11 & 17 & Lathière et al. (2006) & ORCHIDEE & $50^{\circ} \mathrm{E}$ \\
10 & 9 & 4.6 & Simpson et al. (1999) & EMEP & $\mathrm{d}$ \\
7.5 & 7.5 & 4.5 & Simpson et al. (1995) & EMEP & $\mathrm{d}$ \\
29 & & Lübkert and Schöpp (1989) & Forest VOC Inv. & $42^{\circ} \mathrm{E}$ \\
\hline
\end{tabular}

a Total amount of BVOC emissions from all land use types.

b BVOC emissions from forests only.

c Global BVOC inventory. The estimates are from model simulations using the global inventory.

${ }^{\mathrm{d}}$ Includes the European part of the former USSR.

to November, SON). Agriculture BVOC emissions sum up to $113 \mathrm{Gg}$ per year and this is $50 \%$ higher than the estimate of Simpson et al. (1999) for pastures and crops in the United Kingdom (i.e. Great Britain excluding Northern Ireland). This discrepancy may be an indication for the importance of using crop-specific land use information together with cropspecific growing seasons. In the highly spatially and temporally resolved inventory for Great Britain from Stewart et al. (2003) annual emissions of isoprene and terpenes based on the year 1998 , were found to be $8 \mathrm{Gg}$ and $83 \mathrm{Gg}$, respectively. Their estimate of $8 \mathrm{Gg} \mathrm{yr}^{-1}$ isoprene emissions is seven times less than the isoprene emission given in Simpson et al. (1999) for the United Kingdom and four times less than our estimate of $32 \mathrm{Gg} \mathrm{yr}^{-1}$. Stewart et al. (2003) concluded that the standard emission potential attributed to the isoprene emissions from Sitka spruce (Picea sitchensis) is crucial in determining isoprene emissions from Great Britain.

In Finland forests clearly are the dominant BVOC source. Forest emissions increase by a factor of 5 between MAM and JJA. Isoprene contributes only $12 \%$ to the JJA forest emissions in Finland. BVOC emissions in Finland are $8 \mathrm{Gg} \mathrm{yr}^{-1}$ from agriculture crops and $404 \mathrm{Gg} \mathrm{yr}^{-1}$ from forest, and thus close to the estimate given by Simpson et al. (1999). Other land use BVOC emissions are much higher than in the inventory from Simpson et al. (1999), indicating that wetlands, moors and heathlands are probably more important emitters than thought previously. The important role of wetlands for isoprene emissions in Finland was confirmed in the recent inventory study by Tarvainen et al. (2007). Our annual forest BVOC and isoprene emission amounts are more than two times higher than the respective estimates of $146 \mathrm{Gg}$ BVOC and $15 \mathrm{Gg}$ isoprene for boreal forests in Finland given by Tarvainen et al. (2007) for the year 1997. One reason for the lower forest emissions in this inventory may be the use of lower isoprene and monoterpene standard emission potentials for Norway spruce (Picea abies).

BVOC emissions from Germany mainly originate from agriculture and forests with a respective contribution of $35 \%$ and $62 \%$. Total BVOC emissions in JJA are a factor of 3 higher than in MAM. Isoprene contributes to the JJA BVOC emissions from agriculture and forests with $9 \%$ and $33 \%$, respectively. The contribution of isoprene to the yearly averaged BVOC emissions from forests is found to be $28 \%$ for Germany. Isoprene emissions from forests sum up to $62 \mathrm{Gg}$ per year, and are $45 \%$ lower than German isoprene forest emissions given by Simpson et al. (1999). Our forest isoprene emissions for Germany are in good agreement with the average isoprene forest emissions of $40 \mathrm{Gg} \mathrm{yr}^{-1}$ for the decade 1994-2003 found in the German forest BVOC inventory by Smiatek and Steinbrecher (2006). Our forest BVOC emission estimate of $217 \mathrm{Gg} \mathrm{yr}^{-1}$ is lower than the estimate of $377 \mathrm{Gg} \mathrm{yr}^{-1}$ by Simpson et al. (1999) and the estimate of $366 \mathrm{Gg} \mathrm{yr}^{-1}$ by Smiatek and Steinbrecher (2006). In our inventory, agriculture is found to be an important source, emitting $123 \mathrm{Gg} \mathrm{yr}^{-1}$ BVOC. This amount exceeds the emission estimate from Simpson et al. (1999) for crops in Germany by a factor of two.

\subsection{Comparison with other European estimates}

The results obtained in our work are compared to other estimates of European BVOC emissions. A list of previous estimates of isoprene and total BVOC emissions for Europe is provided in Table 8 . The most recent of the listed estimates is from Steinbrecher et al. (2009), the NatAir project inventory of VOC emission from natural and semi-natural vegetation. 
The NatAir model domain has almost the same extent as the Pan-European domain of our study. Steinbrecher et al. (2009) were mainly interested in natural emissions of BVOC and therefore agriculture emissions were estimated using the general agriculture classes from the CLC/GLC2000 mosaic and they applied a default parameterisation for agriculture emission factors and foliar biomass densities. For EU27 they made use of the forest tree species dataset from the Tree Species Map (Köble and Seufert, 2002) while for all other parts of the European domain and for all other land use types the general land use classes from CLC/GLC2000 were used. The use of general land use classes instead of plant-specific land use data can lead to large inaccuracies and will be discussed in Sect. 4.1. The NatAir study calculates European emissions based on the years 1997, 2000, 2001 and 2003 to address inter-annual variability of emissions. The years 1997 and 2002 were fairly cold years while 2001 and in particular 2003 were relatively warm years. Their estimate of annual total BVOC emissions amounts to $20 \mathrm{Tg}$ for the average of the four years, with a variation from $15-25 \mathrm{Tg}$ for the particular years. The lowest NatAir estimate of $15 \mathrm{Tg}$ is about $2-3 \mathrm{Tg}$ higher than our estimate and the earlier estimate given by Simpson et al. (1999). From the average total NatAir amount of $20 \mathrm{Tg}$ BVOC emissions, $12 \mathrm{Tg}$ are estimated to originate from forests and $8 \mathrm{Tg}$ from other land uses, including agriculture. Non-forest emissions obtained in our work sum up to $5 \mathrm{Tg}$ and are lower than the average NatAir non-forest emissions.

Forest emissions of our estimate are $45 \%$ lower than the NatAir estimate. Due to the use of the identical forest tree species information (forest cover database and standard emission factors), the emissions from forests should be comparable. A possible reason for the deviation may be the higher temperatures in the selected years of the NatAir study. Forest emissions obtained in our work are very similar to the early estimate of Lübkert and Schöpp (1989). These authors used a very simple model for calculating forest emission based on altitude-resolved forest cover data and spatially and temporally interpolated temperature data. They used only one temperature-dependent emission factor for each deciduous and coniferous trees. These emission factors were different for day and for night. The good agreement with our work indicates that the use of accurate forest data is probably more important for the prediction of forest emissions than the application of a great amount of detail to describe the short-term variation of emissions.

The $4 \mathrm{Tg}$ lower annual OVOC emission estimate for Europe of our study compared to the NatAir estimate can be explained by the different aggregation procedure applied in this work and the applied growing season concept which includes detailed growing season lengths for seven different agricultural crops. The same default standard emission factor for OVOC as in the NatAir inventory was applied in this work and also the land use information is based on the same database. In contrast to the NatAir inventory plant- specific agriculture land use data was employed in the presented work.

Our isoprene emission estimate of $3.5 \mathrm{Tg}$ is about $1 \mathrm{Tg}$ lower than previous estimates for Europe that are based on European bottom-up inventories (Simpson et al., 1995, 1999; Steinbrecher et al., 2009). Likely reasons for the lower isoprene emissions in our inventory include the use of different meteorology data, lower temperatures and light abundance in the selected years, and the application of a biomass correction factor for main tree species. The impact of the bioclimatic correction factor on our European emission estimate will be discussed in Sect. 4.4.

We compared our estimate for Europe with the estimate from global bottom-up isoprene emission inventories using the canopy environment model as described in Sect. 2.4. Global inventories are known to incorporate a lower degree of detail and are commonly used for global estimates of vegetation emissions. In the scope of this work, simulations with two different global isoprene inventories, the GEIA inventory (Guenther et al., 1995) and the recent MEGAN (Guenther et al., 2006), were performed for the years 2002 and 2003. Meteorological surface data was used with the same horizontal resolution as applied for the presented emission estimate. Using the G97 algorithm together with the GEIA isoprene emission inventory resulted in $10 \mathrm{Tg}$ isoprene emissions per year in Europe, while using the MEGAN algorithm together with the global MEGAN input variables resulted in a lower estimate of $4.5 \mathrm{Tg}$.

The difference of the annual amounts of European isoprene emissions between the two global inventories and the presented plant-specific European emission inventory can be attributed to the use of plant-specific foliar biomass density distributions and isoprene standard emission factor distributions for Europe in our inventory. The annual European isoprene emissions calculated with the global isoprene emission inventory of the MEGAN model (Guenther et al., 2006) are in exact agreement with the European inventory of Simpson et al. (1999) which is likely due to their use of similar plant species distribution data. In general, the MEGAN inventory agrees well with recent European bottom-up estimates (Table 8), while the former GEIA inventory overestimates isoprene emissions in Europe by more than factor 2 compared to European bottom-up inventories. It has been recognized that the minimum level of uncertainty in global biogenic emission estimates is a factor of 3 (Guenther et al., 1995) and can be higher for regional estimates.

\section{Discussion of uncertainties}

In this chapter the uncertainties of biogenic emission due to inaccurate land use information, BVOC standard emission factors, biomass densities and driving variables are discussed. 


\subsection{General land use and default values}

Global inventories of BVOC emissions are built on general land use classes, like ecosystems (e.g. boreal conifers in the GEIA inventory, Guenther et al., 1995) or plant distribution functions (e.g. broadleaf evergreen trees in the MEGAN inventory, Guenther et al., 2006) or vegetation classes (CLC/GLC2000 mosaic). The MEGAN inventory uses geo-referenced plant species distribution data where these are available (e.g. in the US, in Canada and in Australia) while more generic data is used in other world regions. In Europe, national tree data provided by Simpson et al. (1999) was employed in MEGAN to derive the plant distribution functions for forest land use.

The generalized way to define the geographic distribution of land use classes and the often insufficient differentiation of vegetation types can lead to a systematic bias in biogenic emission inventories.

In the presented inventory, we relied on plant-specific georeferenced land use information for all the parts of Europe where this kind of data is available. However, for most Eastern European countries and especially for Russia we had to use the general vegetation classes of CLC/GLC2000. Standard emission factors and foliar biomass densities for agriculture and forest CLC/GLC2000 landcover classes were derived from the predominant plant species in each class. The vegetation class foliar biomass density $d_{l}$ is thus obtained from the weighted foliar biomass densities of major tree or crop species that contribute to the vegetation composition of the respective class. However, vegetation classes often cover large geographic regions and plant composition of classes may not be representative of the complete geographic extent. For example, the Global Landcover (GLC) vegetation class Needleleaved Evergreen Forest covers large parts of boreal Northern Russia. The GLC Northern Eurasia (v4.0) classifies this forest to consist mainly of Picea, Abies and Pinus trees. This forest type covers large areas by $100 \%$, ignoring that there are actually other land use types like wetlands in these areas. This leads to inconsistencies of the calculated foliar biomass density and becomes clearly visible at the Russian border to Finland (see Fig. A1b, Electronic Supplement: http://www.biogeosciences.net/6/1059/ 2009/bg-6-1059-2009-supplement.pdf). This country border marks the border between plant-specific land use classes and the general land use classes from GLC.

\subsection{Standard emission factors}

The use of default values for standard emission factors for the OVOC and SQT classes tend to produce inaccurate results. Default values for these compound groups are applied in this inventory to give an European estimate in spite of missing measured data for most plant species. There is a clear need for future research on the chemical speciation and emission factors of oxygenated VOC for different crop and tree species. Oxygenated VOC are included in our emission inventory using the temperature dependent monoterpene emission algorithm. However, daily cycles of ambient concentrations of methanol, acetone and acetaldehyde, indicate that OVOC emissions are governed by different processes (Rinne et al., 2007) and should respond differently to light, drought or herbivore attack.

Biogenic emissions among plants of the same species or even among parts of the same plant may vary considerably. Funk et al. (2005) found that under normal climate conditions, isoprene emissions from different red oak trees within a single stand differed by a factor of two. Komenda and Koppmann (2002) reported that monoterpenes emission rates measured from two different branches of the same Scots pine tree differed by a factor of two. Monoterpene emission rates among different Scots pine trees of the same age and origin, growing under similar conditions, even varied by more than one order of magnitude (Komenda and Koppmann, 2002). In the BEMA (Biogenic Emissions in the Mediterranean Area) campaigns, 1993-1994, a high variability of standard emission factors was observed for Quercus ilex and Pinus pinea, probably due to different stages of leaf development, tree age, habitat and light adaption (Seufert et al., 1997).

\subsection{Emissions from agriculture}

Monoterpene emissions from agriculture are highly uncertain since studies on agricultural crops are scarce and the applied standard emission potentials are probably biased by measurements at higher-than-ambient temperatures. To date, the investigation of König et al. (1995) is the only comprehensive work on BVOC emissions from agricultural crops in Europe (see Table 7 in Kesselmeier and Staudt, 1999). Annual monoterpene emissions from agriculture in our inventory sum up to about $0.9 \mathrm{Tg}$ and are lower than the estimate of about $2 \mathrm{Tg}$ given by Steinbrecher et al. (2009) for other landuse. It should however be considered that the definition of other landuse in the NatAir inventory includes agriculture and other land use types (grassland, wetland, shrubs etc.). Agriculture is an important source for OVOCs and monoterpenes in our inventory, comprising about $35 \%$ of the total annual OVOC and monoterpene emissions.

In our inventory, OVOC due to the high default emission factor of $2.0 \mu \mathrm{g} \mathrm{g}_{\mathrm{DW}}^{-1} \mathrm{~h}^{-1}$ predominantly contributes to agriculture BVOC emissions. Until now, it is unclear if the main crops like wheat and other cereals emit OVOC in significant quantities. For example, Winer et al. (1992) detected no oxygenated VOC emissions from wheat. König et al. (1995) have found that total plant BVOC emission of oilseed rape (normalised to $30^{\circ} \mathrm{C}$ ) were increased by a factor of 2 in the blossoming stage compared to the nonblossoming stage, with increases of factor 4-5 for single monoterpenes like $\alpha$-pinene. Rape cultivations cover 5-10\% of large parts of France, England, Germany and Italy and locally cover about 40\% in Spain (see map in Fig. A4, Electronic Supplement), 
and rape oil is increasingly used as biofuel. These examples illustrate the clear need for more comprehensive studies on BVOC emissions from European agricultural crops.

To quantify the impact of uncertain monoterpene emissions from agriculture for our inventory, we performed a simulation of BVOC emissions for the year 2005 with increased monoterpene emissions from rape cultivations. For this test, the rape standard emission factors for $\mathrm{MT}_{\text {pool }}$ and $\mathrm{MT}_{\text {synt }}$ were increased by a factor of four and set to $2.0 \mu \mathrm{gg}_{\text {DW }}^{-1} \mathrm{~h}^{-1}$. The MT standard emission potentials assigned to all other agriculture crops remained unchanged. Total European monoterpene emissions from agriculture increased by $3 \%$ in this test. Emissions from agriculture in the Atlantic vegetation zone increase by roughly $3 \%$, while the agriculture emissions from the Boreal, Continental and Mediterranean zone are less affected.

The regional impact on BVOC emissions from agriculture depends on the agricultural area fraction covered with rape cultivations. Agriculture BVOC emissions for 2005 increased by $1 \%$ in Spain, $2 \%$ in Great Britain, 3\% in Germany and $4 \%$ in Finland. Rape is cultivated in Southern Finland and increased monoterpene standard emission factors slightly affect the agriculture BVOC emissions. However we note that agriculture plays a minor role in Finland, contributing only $1 \%$ to the total BVOC emissions in Finland.

Though only small changes of agricultural emissions are observed in this test for monoterpene emissions from rape cultivations, we conclude that the estimate of agricultural emissions in Europe is currently biased by the uncertainties in monoterpene and OVOC standard emission potentials for crops.

\subsection{Bioclimatic correction}

To adjust foliar biomass densities for the main tree species and crops in the different vegetation zones we have introduced a bioclimatic correction factor (see Sect. 2.2). European BVOC emissions were recalculated for the year 2005 with the bioclimatic correction set to unity for all trees, crops and general vegetation classes $\left(f_{j}=f_{k}=f_{l}=1\right)$, in order to test the sensitivity of emissions towards this parameter. The total emitted European amount of BVOC from this simulation is only $3 \%$ higher than the amount calculated with bioclimatic correction factors according to Tables 1 and 2. For single BVOC classes annual European emissions changed less than $5 \%$.

However this factor is important when estimating BVOC emissions from major vegetation zones and countries within Europe. In the Atlantic vegetation zone, total BVOC emissions for 2005 are $17 \%$ higher and agriculture BVOC emissions are $26 \%$ higher when considering the bioclimate correction of foliar biomass densities. This can be explained with bioclimatic correction factors larger than 1 for the main agricultural crops in the Atlantic vegetation zone, leading to elevated foliar biomass densities in Western Europe (see Fig. A1a, Electronic Supplement: http://www.biogeosciences.net/6/1059/ 2009/bg-6-1059-2009-supplement.pdf). This is also reflected in the emissions from Great Britain, which are higher by $12 \%$. In the Mediterranean vegetation zone, total BVOC emissions for the year 2005 are 5\% lower when considering the bioclimate correction of foliar biomass densities. Agriculture BVOC emissions are $20 \%$ lower, indicating that bioclimatic correction factors below 1 for main crops in the Mediterranean zone are the cause for the discrepancy. In Spain, emissions are only $2 \%$ lower due to the minor importance of agriculture emissions. In the Boreal vegetation zone, BVOC emissions for 2005 are only $1 \%$ lower when taking into account the bioclimatic correction of foliar biomass densities. Though bioclimatic correction factors for the main tree species in the Boreal zone are less than 1, the impact on total BVOC emission is limited. As expected, BVOC emissions of Germany remained unchanged, since this country is within the Continental zone which is used as a reference zone for defining the bioclimatic correction.

It can thus be concluded, that the introduction of corrected foliar biomass densities has a significant impact on regional BVOC emission estimates, while, on the European scale, the annual and the monthly emitted European amount of BVOC is only marginally influenced by the correction for bioclimatic zones. However, it can not be excluded that a less conservative and more realistic application of bioclimatic corrections which relies on a larger amount of tree specific biomass data may change this picture. For individual bioclimatic zones the bioclimatic correction factors for tree species rely on very few litterfall data records (e.g. Pinus sylvestris in the Atlantic and Mediterranean zone). Further exploitation of the biomass compartment databases like the one from AFOLU (see Sect. 2.2) data will help to improve the situation in future.

\subsection{Driving variables}

The commonly observed temperature and light dependence of BVOC emissions seems to hold also at high latitudes in boreal and subarctic regions. It has been shown that the light and temperature dependent parameterization of BVOC emissions is applicable to northern boreal environmental conditions (Rinne et al., 2000; Tarvainen et al., 2005, 2007; Hakola et al., 2006). Tiiva et al. (2008) demonstrated the increase of isoprene emission due to temperature increase in subarctic heath land. The effect was found to be a direct response to increased air temperature, which indicates a rapid physiological effect of current temperature on isoprene production as outlined in Lerdau and Gray (2003). The mean isoprene emissions from subarctic heath land were comparable to the emissions found earlier from a subarctic peatland ecosystem (Tiiva et al., 2007). The emissions from the subarctic peatland increased 10-fold during an exceptionally warming 
period, again giving evidence for the validity of the proposed temperature- and light-dependence (Tiiva et al., 2007).

Emissions are not only dependent on exogenous driving variables like temperature and light but dependent also on endogenous parameters such as the developmental state of the leaves. While young leaves of deciduous trees begin photosynthesis shortly after budbreak, emission of isoprene only starts after a retardation of several days. On the other hand, older leaves produce isoprene less efficiently. Reduced emissions in autumn have been found to be correlated with the activity of some terpene synthases (Lehning et al., 1999) and a seasonality for monoterpene synthase activity has been observed for Quercus ilex (Fischbach et al., 2002). Based on long term laboratory experiments with Pinus pinea trees, Staudt et al. (2000) developed an algorithm to adjust for seasonality that was proven to fit very well with observations in the field, where monoterpene emission factors of this species varied between 2.8 and $15 \mu \mathrm{g} \mathrm{g}_{\mathrm{DW}}^{-1} \mathrm{~h}^{-1}$ during the course of the year (Staudt et al., 1997). The algorithm of Staudt et al. (2000) has been applied in this work to determine the (longterm) seasonal variation of emissions from deciduous and evergreen vegetation.

Soil water availability represents an important environmental constraint under Mediterranean conditions. In the Mediterranean area, summer months are characterized by the highest annual temperatures and radiation levels which occur simultaneously with the most severe drought conditions. Under drought conditions, plants will experience increasing water deficit stress. This affects emissions of different VOC classes in different ways, depending on whether BVOCs are emitted from current photosynthesis like in case of isoprene and $\mathrm{MT}_{\text {synt }}$ or from reservoir pools $\left(\mathrm{MT}_{\text {pool }}\right)$. Pegaro et al. (2004) showed for Quercus virginiana Mill. that isoprene emissions remained stable during the first eight days of water withholding and declined thereafter. SQT emissions from orange trees are reported to be strongly reduced under severe water deficit, while not being influenced under mild water stress conditions (Hansen and Seufert, 2003). Results obtained by Ormeño et al. (2007) indicate that emissions of sesquiterpenes are replaced by monoterpenes when drought is prolonged. They showed that water stress has a positive effect on monoterpene emissions of Pinus halepensis and Cistus albidus which are both $\mathrm{MT}_{\text {pool }}$ emitter. Monoterpene emissions of water stressed Rosmarinus officinalis remained stable (Ormeño et al., 2007). Species like Cistus and Rosmarinus contain aromatic oils in external glandular hairs which allow monoterpene emissions to be maintained during drought conditions until the reservoir gets depleted. In case of BVOC emission from current photosynthesis, a severe drought with prolonged reduction of photosynthesis may reduce emission due to a depletion of resources (Bertin and Staudt, 1996).

At ambient ozone levels, reactive terpenoids like ocimene, linalool, limonene and most sesquiterpenes are partly removed within the canopy or within leaf or branch cham- bers, if flushed with ambient air (Seufert et al., 1997). Above canopy fluxes are much smaller than emissions derived from branch enclosures due to losses by deposition and oxidation within the canopy (Ciccioli et al., 1999). Within-canopy gasphase reactions of ozone with monoterpenes, sesquiterpenes and other oxygenated hydrocarbons with short chemical lifetimes will lead to SOA formation inside the canopy. The estimated fluxes of our inventory thus are more representative of the sum of parent hydrocarbon, its oxidations products and particles released to the atmosphere. Transformation and deposition losses should thus be taken into account in future BVOC emission inventories.

Uncertainties of the main driving variables temperature, solar radiation and leaf area index have shown to result in emission estimates that differ more than a factor of three for specific times and locations (Guenther et al., 2006). The additional driving factors mentioned above can potentially be addressed in a canopy environment model of the MEGAN type. The plant-specific European inventory of foliar biomass densities developed in this work can be used together with less biased emission factors and with a more sophisticated canopy emission model in the future to obtain more accurate BVOC emission estimates for Europe with a high temporal and spatial resolution.

\section{Conclusions}

Agriculture land use types are identified in our study as an important source for OVOCs and monoterpenes. However, large uncertainties remain for the OVOC class since chemically speciated measurements are lacking for most plant species. Monoterpene emissions from agriculture are highly uncertain because the estimate relies on scarce literature data available for crops in Europe and on early screening studies. The small number of available studies on agriculture emissions is a serious limitation of current BVOC emission inventories for Europe. In our European inventory, for the first time, crop-specific land use information was employed together with crop-specific standard emission factors wherever applicable. Further work on agriculture emissions should be carried out to check the validity of standard emission factors for monoterpenes and OVOC. In view of the discussion on the increased use of biofuels in Europe it will be of great importance to give a more accurate estimate of BVOC emissions from agriculture crops cultivated for biofuel production.

The presented emission inventory serves as an improved basis - especially with regard to the plant-specific land use data - for estimating BVOC emissions in Europe. In contrast to previous BVOC emission inventories, a bioclimatic correction factor was introduced to correct the foliar biomass densities for the different plant growth conditions that can be found in Pan-Europe. This leads to reduced BVOC emissions from agriculture in the Mediterranean while emissions 
from agriculture in Western Europe are increased compared to an estimate that does not consider the influence of different growth conditions on the foliar biomass density. Forests are found to be the largest source for BVOCs released to the atmosphere in Europe and therefore an accurate estimate of the foliar biomass density for the main tree species is essential for the correct prediction of emissions.

To improve predictions of European air quality it is important to use BVOC emissions that are as accurate as possible since the importance of anthropogenic VOC as precursors of photochemically produced ozone may decline due to pollution abatement strategies.

The databases of foliar biomass densities, standard emission potentials, and resulting emissions are created in HDF format to facilitate the implementation into atmospheric transport models. The databases can be obtained upon request from the authors and are made available on the AFOLU portal (http://afoludata.jrc.ec.europa.eu/index.php/ dataset/info/121). To access the dataset on the web portal it is necessary to register (http://afoludata.jrc.ec.europa.eu/index. php/new_user).

Resulting European emission fields can be used in the future to investigate secondary particle formation from isoprene, monoterpenes and sesquiterpenes with regional or global chemistry transport models.

Acknowledgements. We thank Tracy Durrant for the Forest Focus litterfall data, Maurizio Teobaldelli for retrieving foliar biomass data from the AFOLU database, and Laurens Ganzeveld for providing the radiation code. We also thank Jürgen Wildt for constructive discussion and Frank Dentener for helpful comments.

Edited by: C. Spirig

\section{References}

Andreae, M. O. and Crutzen, P.: Atmospheric aerosols: Biogeochemical sources and role in atmospheric chemistry, Science, 276, 1052-1088, 1997.

Andreani-Aksoyoglu, S. and Keller, J.: Estimates of monoterpene and isoprene emissions from the forests in Switzerland, J. Atmos. Chem., 20(1), 71-87, 1995.

Andryukov, V. P. and Timofeev, A. B.: Assessment of volatile organic compounds emissions from natural sources in Europe, Contribution to the 4th ECE Task Force on Volatile Organic Comounds, Schweitzingen, Federal Republic of Germany, 30 May2 June 1989.

Arey, J., Winer, A. M., Atkinson, R., Aschmann, S. M., Long, W. D., Morrison, C. L., and Olszyk, D. M.: Terpenes emitted from agricultural species found in California's Central Valley, J. Geophys. Res., 96(D5), 9329-9336, 1991.

Atkinson, R. and Arey, J.: Gas-phase tropospheric chemistry of biogenic volatile organic compounds: A review, Atmos. Environ., 31(S2), 197-219, 2003.

Bertin, N. and Staudt, M.: Effect of water stress on monoterpene emissions from young potted holm oak (Quercus ilex L.) trees, Oecologia, 107(4), 456-462, 1996.
Bertin, N., Staudt, M., Hansen, U., Seufert, G., Ciccioli, P., Foster, P., Fugit, J.-L., and Torres, L.: The BEMA-project: Diurnal and seasonal course of monoterpene emissions from Quercus ilex (L.) under natural conditions - application of light and temperature algorithms, Atmos. Environ., 31(S1), 135-144, 1997.

Boissard, C., Chervier, F., and Dutot, A. L.: Assessment of high (diurnal) to low (seasonal) frequency variations of isoprene emission rates using a neural network approach, Atmos. Chem. Phys., 8, 2089-2101, 2008, http://www.atmos-chem-phys.net/8/2089/2008/.

Bonn, B. and Moortgat, G. K.: Sesquiterpene ozonolysis: Origin of atmospheric new particle formation from biogenic hydrocarbons, Geophys. Res. Lett., 30(11), 1585, doi:10.1029/2003GL017000, 2003.

Bouraoui, F. and Aloe, A.: European Agrochemicals Geospatial Loss Estimator: Model Development and Applications. European Commission, Joint Research Centre, Institute for Environment and Sustainability, ISBN 978-92-79-05053-4, p. 107, 2007.

Ciccioli, P., Brancaleoni, E., Frattoni, M., Di Palo, V., Valentini, R., Tirone, G., Seufert, G., Bertin, N., Hansen, U., Csiky, O., Lenz, R., and Sharma, M.: Emission of reactive terpene compounds from orange orchards and their removal by within-canopy processes, J. Geophys. Res., 104(D7), 8077-8094, 1999.

Claeys, M., Graham, B., Vas, G., Wang, W., Vermeylen, R., Pashynska, V., Cafmeyer, J., Guyon, P., Andreae, M. O., Artaxo, P., and Maenhaut, W.: Formation of secondary organic aerosols through photooxidation of isoprene, Science, 303, 1173-1176, 2004.

Csiky, O. and Seufert, G.: Terpenoid emissions of Mediterranean oaks and their relation to taxonomy, Ecol. Appl., 9(4), 11381146, 1999.

Duhl, T. R., Helmig, D., and Guenther, A.: Sesquiterpene emissions from vegetation: a review, Biogeosciences, 5, 761-777, 2008, http://www.biogeosciences.net/5/761/2008/.

EMEP/CORINAIR: Atmospheric Emission Inventory Guidebook, Second Edition, Copenhagen: European Environment Agency, online available at: http://reports.eea.europa.eu/ EMEPCORINAIR/en/page007.html, 1999.

Dindorf, T., Kuhn, U., Ganzeveld, L., Schebske, G., Ciccioli, P., Holzke, C., Köble, R., Seufert, G., and Kesselmeier, J.: Significant light and temperature dependent monoterpene emissions from European beech (Fagus sylvatica L.) and their potential impact on the European volatile organic compound budget, J. Geophys. Res., 111, D16305, doi:10.1029/2005JD006751, 2006.

European Environment Agency: European Topic Center on Biological Diversity, Under contract with the European Environment Agency, Indicative Map of European Biogeographical Regions, 2005, Version 7, online available at: http://www.eea.europa.eu, EEA, Copenhagen, 2007.

FAO Statistics Division, FAOSTAT: Agriculture Organization of the United Nations (FAO), online available at: http://faostat.fao.org, 2007.

Fischbach, R., Staudt, M., Zimmer, I., Rambal, S., and Schnitzler, J.-P.: Seasonal pattern of monoterpene synthase activities in leaves of the evergreen tree Quercus ilex L., Physiol. Plantarum, 114, 354-360, 2002.

Funk, J. F., Jones, C. G., Gray, D. W., Throop, H. L., Hyatt, L. A., and Lerdau, M. T.: Variation in isoprene emission from Quercus rubra: Sources, causes, and consequences for estimating fluxes, J. Geophys. Res., 110, D04301, doi:10.1029/2004JD005229, 
2005.

Geron, C. D., Guenther, A. B., and Pierce, T. E.: An improved model for estimating emissions of volatile organic compounds from forests in the eastern United States, J. Geophys. Res., 99(D6), 12773-12791, 1994.

Griffin, R. J., Cocker, D. R., Seinfeld, J. H., and Dabdub, D.: Estimate of global atmospheric organic aerosol from oxidation of biogenic hydrocarbons, Geophys. Res. Lett., 26, 2721-2724, 1999.

Guenther, A.: Seasonal and spatial variations in natural volatile organic compound emissions, Ecol. Appl., 7, 34-45, 1997.

Guenther, A.: The contribution of reactive carbon emissions from vegetation to the carbon balance of terrestrial ecosystems, Chemosphere, 49, 837-844, 2002.

Guenther, A., Zimmerman, P. R., Harley, P. C., Monson, R. K., and Fall, R.: Isoprene and monoterpene emission rate variability: Model evaluations and sensitvity analyses, J. Geophys. Res., 98(D7), 12609-12617, 1993.

Guenther, A., Zimmerman, P., and Wildermuth, M.: Natural volatile organic compound emission rate estimates for U.S. woodland landscapes, Atmos. Environ., 28(6), 1197-1210, 1994.

Guenther, A., Hewitt, C. N., Erickson, D., Fall, R., Geron, C., Graedel, T., Harley, P., Klinger, L., Lerdau, M., McKay, W. A., Pierce, T., Scholes, B., Steinbrecher, R., Tallamraju, R., Taylor, J., and Zimmerman, P.: A global model of natural volatile organic compound emissions, J. Geophys. Res., 100, 9973-8892, 1995.

Guenther, A., Karl, T., Harley, P., Wiedinmyer, C., Palmer, P. I., and Geron, C.: Estimates of global terrestrial isoprene emissions using MEGAN (Model of Emissions of Gases and Aerosols from Nature), Atmos. Chem. Phys., 6, 3181-3210, 2006, http://www.atmos-chem-phys.net/6/3181/2006/.

Hakola, H., Rinne, J., and Laurila, T.: The hydrocarbon emission rates of Tea-Leafed Willow (Salix phylicifolia), Silver Birch (Betula pendula) and European Aspen (Populus tremula), Atmos. Environ., 32(10), 1825-1833, 1998.

Hakola, H., Laurila, T., Lindfors, V., Hellén, H., Gaman, A., and Rinne, J.: Variation of the VOC emission rates of birch species during the growing season, Boreal Environ. Res., 6, 237-249, 2001.

Hakola, H., Tarvainen, V., Bäck, J., Ranta, H., Bonn, B., Rinne, J., and Kulmala, M.: Seasonal variation of mono- and sesquiterpene emission rates of Scots pine, Biogeosciences, 3, 93-101, 2006, http://www.biogeosciences.net/3/93/2006/.

Hansen, U. and Seufert, G.: Temperature and light dependence of beta-caryophyllene emission rates, J. Geophys. Res., 108(D24), 4801, doi:10.1029/2003JD003853, 2003.

Helmig, D., Ortega, J., Duhl, T., Tanner, D., Guenther, A., Harley, P., Wiedinmyer, C., Milford, J., and Sakulyanontvittaya, T.: Sesquiterpene emissions from pine trees - Identifications, emission rates and flux estimates for the contiguous United States, Environ. Sci. Technol., 41, 1545-1553, 2007.

Hoffmann, T., Odum, J. R., Bowman, F., Collins, D., Klockow, D., Flagan, R. C., and Seinfeld, J. H.: Formation of organic aerosol from the oxidation of biogenic hydrocarbons, J. Atmos. Chem., 26, 189-222, 1997.

Holland, E. A., Post, W. M., Matthews, E. G., Sulzman, J., Staufer, R., and Krankina, O.: Global Patterns of Litterfall and Litter Pool Carbon and Nutrients, Data set available online at: http://daac.ornl.gov/ from Oak Ridge National Laboratory Distributed Active Archive Center, Oak Ridge, Tennessee, USA, 2005.

Jaoui, M., Leungsakul, S., and Kamens, R. M.: Gas and particle distribution from the reaction of $\beta$-caryophyllene with ozone, J. Atmos. Chem., 45, 261-287, 2003.

Kavouras, I. G., Mihalopoulos, N., and Stephanou, E. G.: Formation of atmospheric particles from organic acids produced by forests, Nature, 395, 683-686, 1998.

Kerminen, V. M., Lihavainen, H., Komppula, M., Viisanen, Y., and Kulmala, M.: Direct observational evidence linking atmospheric aerosol formation and cloud droplet activation, Geophys. Res. Lett., 32, L14803, doi:10.1029/2005GL023130, 2005.

Kesselmeier, J. and Staudt, M.: Biogenic Volatile Organic Compounds (VOC): An overview on emission, physiology and ecology, J. Atmos. Chem., 33, 23-88, 1999.

Kesselmeier, J., Ciccioli, P., Kuhn, U., Stefani, P., Biesenthal, T., Rottenberger, S., Wolf, A., Vitullo, M., Valentini, R., Nobre, A., Kabat, P., and Andreae, M. O.: Volatile organic compound emissions in relation to plant carbon fixation and the terrestrial carbon budget, Global Biogeochem. Cy. 16(4), 1126, doi:10.1029/2001GB001813, 2002.

Komenda, M. and Koppmann, R.: Monoterpene emissions from Scots Pine (Pinus sylvestris): Field studies of emission rate variabilities, J. Geophys. Res., 107(D13), 4161, doi:10.1029/2001JD000691, 2002.

Kourtchev, I., Ruuskanen, T., Maenhaut, W., Kulmala, M., and Claeys, M.: Observation of 2-methyltetrols and related photooxidation products of isoprene in boreal forest aerosols from Hyytiälä, Finland, Atmos. Chem. Phys., 5, 2761-2770, 2005, http://www.atmos-chem-phys.net/5/2761/2005/.

Kroll, J. H., Ng N. L, Murphy, S. M., Flagan, R. C., and Seinfeld, J. H.: Secondary aerosol from isoprene photooxidation under high- $\mathrm{NO}_{\mathrm{x}}$ conditions; Geophys. Res. Lett., 32, L18808, doi:10.1029/2005GL023637, 2005.

Köble, R. and Seufert, G.: Novel maps for forest tree species in Europe, in: Proceedings of the 8th European Symposium on the Physico-Chemical Behaviour of Atmospheric Pollutants, edited by: Hjorth, J., Raes, F., and Angeletti, G., published on CDROM by The Institute of Environment and Sustainability, JRC of Ispra, DG Research, European Commission, Ispra, Italy, Paper TP 35, online available at: http://ies.jrc.ec.europa.eu/Units/ cc/events/torino2001/torinocd/, 2002.

Köble, R.: Land use/cover map, in: NATAIR, Improving and applying methods for the calculation of natural and biogenic emissions and assessment of impacts to the air quality, European Commission - Sixth Framework Programme, Specific Targeted Research or Innovation Project, Proposal No.: 513699, Final activity report, Chapter 4.2, online available at: http://natair.ier. uni-stuttgart.de/, 2007.

König, G., Brunda, M., Puxbaum, H., Hewitt, C. N., and Duckham, S. C.: Relative contribution of oxygenated hydrocarbons to the total biogenic VOC emissions of selected Mid-European plant species, Atmos. Environ., 29(8), 861-874, 1995.

Lathière, J., Hauglustaine, D. A., Friend, A. D., De NobletDucoudré, N., Viovy, N., and Folberth, G. A.: Impact of climate variability and land use changes on global biogenic volatile organic compound emissions, Atmos. Chem. Phys., 6, 2129-2146, 2006, 
http://www.atmos-chem-phys.net/6/2129/2006/.

Lehning, A., Zimmer, I., Steinbrecher, R., Brüggemann, N., and Schnitzler, J. P.: Isoprene synthase activity and its relation to isoprene emission in Quercus robur L. leaves, Plant Cell Environ., 22, 494-505, 1999.

Leip, A., Marchi, G., Koeble, R., Kempen, M., Britz, W., and Li, C.: Linking an economic model for European agriculture with a mechanistic model to estimate nitrogen and carbon losses from arable soils in Europe, Biogeosciences, 5, 73-94, 2008, http://www.biogeosciences.net/5/73/2008/.

Lenz, R., Köble, R., and Seufert, G.: Species-based mapping of biogenic emissions from European forests - Case study Italy and France in: Proceedings of the 8th European Symposium on the Physico-Chemical Behaviour of Atmospheric Pollutants, edited by: Hjorth, J., Raes, F., and Angeletti, G., published on CDROM by The Institute of Environment and Sustainability, JRC of Ispra, DG Research, European Commission, Ispra, Italy, Paper TP 15, online available at: http://ies.jrc.ec.europa.eu/Units/ cc/events/torino2001/torinocd/, 2002.

Lerdau, M. and Gray, D.: Ecology and evolution of light-dependent phytogenic volatile organic carbon; New Phytol., 157, 199-211, 2003.

Lübkert, B. and Schöpp, W.: A model to calculate natural VOC emissions from forests in Europe, November 1989, IIASA Working Paper WP-89-082, 1989.

Luyssaert, S., Inglima, I., Jung., M., and co-workers: $\mathrm{CO}_{2}$ balance of boreal, temperate, and tropical forests derived from a global database, Glob. Change Biol., 13, 1-29, doi:10.1111/j.13652486.2007.01439.x, 2007.

Moukhtar, S., Bessagnet, B., Rouil, L., and Simon, V.: Monoterpene emissions from Beech (Fagus sylvatica) in a French forest and impact on secondary pollutants formation at regional scale; Atmos. Environ., 39, 3535-3547, 2005.

Ormeño, E., Mévy, J. P., Vila, B., Bousquet-Mélou, A., Greff, S., Bonin, G. and Fernandez, C.: Water deficit stress induces different monoterpene and sesquiterpene emission changes in Mediterranean species. Relationship between terpene emissions and plant water potential, Chemosphere, 67, 276-284, 2007.

Owen, S. M., Boissard, C., and Hewitt, N.: Volatile organic compounds (VOCs) emitted from 40 Mediterranean plant species: VOC speciation and extrapolation to habitat scale, Atmos. Environ., 35, 5393-5409, 2001.

Pandis, S. N., Paulson, S. E., Seinfeld, J. H., and Flagan, R. C.: Aerosol formation in the photooxidation of isoprene and $\beta$ pinene; Atmos. Environ., 25A, 997-1008, 1991.

Pegoraro, E., Rey, A., Greenberg, J., Harley, P., Grace, J., Malhi, Y., and Guenther, A.: Effect of drought on isoprene emission rates from leaves of Quercus virginiana Mill, Atmos. Environ., 38, 6149-6156, 2004.

Rinne, J., Tuovinen, J.-P., Laurila, T., Hakola, H., Aurela, M., and Hyphén, H.: Measurements of hydrocarbon fluxes by a gradient method above a northern boreal forest, Agr. Forest Meteorol., 102, 25-37, 2000.

Rinne, J., Taipale, R., Markkanen, T., Ruuskanen, T. M., Hellén, H., Kajos, M. K., Vesala, T., and Kulmala, M.: Hydrocarbon fluxes above a Scots pine forest canopy: measurements and modeling, Atmos. Chem. Phys., 7, 3361-3372, 2007, http://www.atmos-chem-phys.net/7/3361/2007/.

Schuh, G., Heiden, A. C., Hoffmann, Th., Kahl, J., Rockel, P.,
Rudolph, J., and Wildt, J.: Emissions of Volatile Organic Compounds from sunflower and beech: Dependence on temperature and light intensity, J. Atmos. Chem., 27, 291-318, 1997.

Seco, R., Penuelas, J., and Filella, I.: Short-chain oxygenated VOCs: emission and uptake by plants and atmospheric sources, sinks, and concentrations, Atmos. Environ., 41, 2477-2499, 2007.

Seinfeld, J. H. and Pandis, S. N.: Atmospheric Chemistry and Physics: From Air Pollution to Climate Change, Wiley, New York, p. 748, 1998.

Seufert, G., Bartzis, J., Bomboi, T., Ciccioli, P., Cieslik, S., Dlugi, R., Foster, P., Hewitt, C. N., Kesselmeier, J., Kotzias, D., Lenz, R., Manes, F., Perez Pastor, R., Steinbrecher, R., Torres, L., Valentini, R., and Versino, B.: The BEMA-project: An overview of the Castelporziano experiments, Atmos. Environ., 31(S1), 517, 1997.

Simpson, D.: Biogenic emissions in Europe 2: Implications for ozone control strategies, J. Geophys. Res., 100(D11), 2289122906, 1995.

Simpson, D., Guenther, A., Hewitt, C. N., and Steinbrecher, R.: Biogenic emissions in Europe, 1. Estimates and uncertainties, J. Geophys. Res., 100(D11), 22875-22890, 1995.

Simpson, D., Winiwarter, W., Börjesson, G., Cinderby, S., Ferreiro, A., Guenther, A., Hewitt, N., Janson, R., Khalil, M. A. K., Owen, S., Pierce, T. E., Puxbaum, H., Shearer, M., Skiba, U., Steinbrecher, R., Tarrasón, L., and Öquist, M. G.: Inventorying emissions from nature in Europe, J. Geophys. Res., 104(D7), 81138152, 1999.

Smiatek, G. and Steinbrecher, R.: Temporal and spatial variation of forest VOC emissions in Germany in the decade 1994-2003, Atmos. Environ., 40(S1), 66-77, 2006.

Staudt, M. and Seufert, G.: Light-dependent emission of monoterpenes by Holm Oak (Quercus ilex L.), Naturwissenschaften, 82, 89-92, 1995.

Staudt, M., Bertin, N., Hansen, U., Seufert, G., Ciccioli, P., Foster, P., Frenzel, B., and Fugit, J.-L.: The BEMA-project: Seasonal and diurnal patterns of monoterpene emissions from Pinus pinea (L.), Atmos. Environ., 31(S1), 145-156, 1997.

Staudt, M., Bertin, N., Frenzel, B., and Seufert, G.: Seasonal variation in amount and composition of monoterpenes emitted by young Pinus pinea trees - implications for emission modeling, J. Atmos. Chem., 35, 77-99, 2000.

Steinbrecher, R., Smiatek, G., Köble, R., Seufert, G., Theloke, J., Hauff, K., Ciccioli, P., Vautard, R., and Curci, G.: Intra- and inter-annual variability of VOC emissions from natural and seminatural vegetation in Europe and neighbouring countries, Atmos. Environ., 43(7), 1380-1391, 2009.

Stewart, H. E., Hewitt, C. N., Bunce, R. G. H., Steinbrecher, R., Smiatek, G., and Schoenemeyer, T.: A highly spatially and temporally resolved inventory for biogenic isoprene and monoterpene emissions: Model description and application to Great Britain, J. Geophys. Res., 108(D20), 4644, doi:10.1029/2002JD002694, 2003.

Teobaldelli M.: The Biomass Compartments database; Data set available online at: http://afoludata.jrc.ec.europa.eu/index.php/ login, 2008.

Tiiva, P., Rinnan, R., Faubert, P., Räsänen, J., Holopainen, T., Kyrö, E., and Holopainen, J. K.: Isoprene emission from subarctic peatland under enhanced UV-B radiation, New Phytol., 176, 346- 
$355,2007$.

Tiiva, P., Faubert, P., Michelsen, A., Holopainen, T., Holopainen, J. K., and Rinnan, R.: Climatic warming increases isoprene emission from a subarctic heath, New Phytol., 180, 853-863, 2008.

Tingey, D. T., Turner, D. P., and Weber, J. A.: Factors controlling the emissions of monoterpenes and other volatile organic compounds, in: Trace Gas Emissions by Plants, edited by: Sharkey, T. D., Holland, E. A., and Mooney, H. A., Academic Press, New York, 93-119, 1990.

Tarvainen, V., Hakola, H., Hellén, H., Bäck, J., Hari, P., and Kulmala, M.: Temperature and light dependence of the VOC emissions of Scots pine, Atmos. Chem. Phys., 5, 989-998, 2005, http://www.atmos-chem-phys.net/5/989/2005/.

Tarvainen, V., Hakola, H., Rinne, J., Hellén, H., and Haapanala, S.: Towards a comprehensive emission inventory from boreal ecosystems, Tellus, 59B, 526-534, 2007.

Tunved, P., Hansson, H.-C., Kerminen, V.-M., Ström, J. , Dal Maso, M., Lihavainen, H., Viisanen, Y., Aalta, P. P., Komppula, M., and Kulmala, M.: High natural aerosol loading over boreal forests, Science, 312, 261-263, 2006.

Veldt, C.: Leaf biomass data for the estimation of biogenic VOC emissions, MTO-TNO report 99-306, 1989.

Veldt, C.: The use of biogenic VOC measurements in emission inventories, TNO-report no. 91-323, 1991.
Vogel, B., Fiedler, F., and Vogel, H.: Influence of topography and biogenic volatile organic compounds emission in the state of Baden-Württemberg on ozone concentrations during episodes of high air temperatures, J. Geophys. Res., 100, 22907-22928, 1995.

Vuorinen, T., Nerg, A.-M., Vapaavuori, E., Jramo, K., and Holopainen, K.: Emission of volatile organic compounds from two silver birch (betula pendula Roth) clones grown under ambient and elevated $\mathrm{CO}_{2}$ and different $\mathrm{O}_{3}$ concentrations, Atmos. Environ., 39, 1185-1197, 2005.

Weiss, A. and Norman, J. M.: Partitioning solar radiation into direct and diffuse, visible and near-infrared components, Agr. Forest Meteorol., 34, 205-213, 1985.

Winer, A. M., Arey, J., Atkinson, R., Aschmann, S. M., Long, W. D., Morrison C. L., and Olszyk, D. M.: Emission rates of organics from vegetation in California's Central Valley, Atmos. Environ., 26A(14), 2647-2659, 1992.

Zhang, P., Anderson, B., Barlow, M., Tan, B., and Myneni, R. B.: Climate-related vegetation characteristics derived from Moderate Resolution Imaging Spectroradiometer (MODIS) leaf area index and normalized difference vegetation index, J. Geophys. Res., 109, D20105, doi:10.1029/2004JD004720, 2004. 